\title{
Optical tweezers in a dusty universe
}

\section{Modeling optical forces for space tweezers applications}

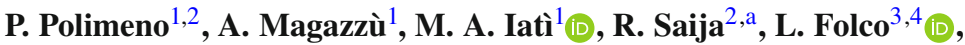 \\ D. Bronte Ciriza ${ }^{1}$, M. G. Donato ${ }^{1}$, A. Foti ${ }^{1}$, P. G. Gucciardi ${ }^{1}$, A. Saidi ${ }^{2}$, \\ C. Cecchi-Pestellini ${ }^{5}$, A. Jimenez Escobar ${ }^{5}$, E. Ammannito ${ }^{6}$, G. Sindoni $^{6}$, I. Bertini ${ }^{7,8}$,

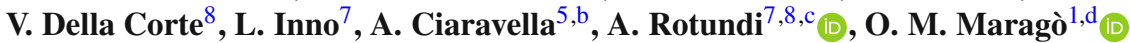 \\ ${ }_{1}^{1}$ CNR-IPCF, Istituto per i Processi Chimico-Fisici, 98158 Messina, Italy \\ 2 Dipartimento di Scienze Matematiche e Informatiche, Scienze Fisiche e Scienze della Terra, Università di \\ Messina, 98166 Messina, Italy \\ 3 Dipartimento di Scienze della Terra, Università di Pisa, Pisa, Italy \\ ${ }^{4}$ CISUP, Centro per l'Integrazione della Strumentazione dell'Università di Pisa, Pisa, Italy \\ 5 INAF Osservatorio Astronomico di Palermo, Palermo, Italy \\ 6 ASI Agenzia Spaziale Italiana, Roma, Italy \\ 7 Dipartimento di Scienze e Tecnologie, Università di Napoli "Parthenope", Napoli, Italy \\ ${ }^{8}$ INAF-IAPS, Istituto di Astrofisica e Planetologia Spaziali, Roma, Italy
}

\begin{abstract}
Optical tweezers are powerful tools based on focused laser beams. They are able to trap, manipulate, and investigate a wide range of microscopic and nanoscopic particles in different media, such as liquids, air, and vacuum. Key applications of this contactless technique have been developed in many fields. Despite this progress, optical trapping applications to planetary exploration are still to be developed. Here we describe how optical tweezers can be used to trap and characterize extraterrestrial particulate matter. In particular, we exploit light scattering theory in the T-matrix formalism to calculate radiation pressure and optical trapping properties of a variety of complex particles of astrophysical interest. Our results open perspectives in the investigation of extraterrestrial particles on our planet, in controlled laboratory experiments, aiming for space tweezers applications: optical tweezers used to trap and characterize dust particles in space or on planetary bodies surface.
\end{abstract}

\section{Optical tweezers}

Mechanical effects of light were first argued by Kepler to explain comet tails [1]. The advent of the laser technology in the 1960s enabled to reproduce these effects on our planet starting a real scientific revolution [2,3]. Optical tweezers [4-7] are tools based on tightly focused laser beams capable to trap, manipulate, and characterize a wide range of microscopic and nanoscopic particles, in liquids, air, and vacuum [6,7]. Since the pioneering work by Ashkin

\footnotetext{
a e-mail: rsaija@unime.it

b e-mail: angela.ciaravella@inaf.it

c e-mail: rotundi@uniparthenope.it (corresponding author)

de-mail: onofrio.marago@cnr.it
} 
[2-4] that led him to the Nobel prize in Physics 2018, key applications of this contactless manipulation technique have been developed in a wide range of fields: from biology, soft matter, and ultra-sensitive spectroscopy to atomic physics, nanoscience, photonics, spectroscopy, and aerosols science [5]. A crucial advancement has been the realization of Raman tweezers, i.e., the coupling of optical tweezers with a Raman spectrometer [8]. This allows the chemical and physical analysis of a trapped particle through its vibrational fingerprints $[9,10]$.

Despite this tremendous experimental progress, the accurate light scattering modeling of optical tweezers, that takes into account particle size, shape, and composition, has been developed only recently $[7,11,12]$. In the limiting cases of particles much smaller or much larger than the laser wavelength, optical forces in optical tweezers can be divided in two components [5]: a gradient force, proportional to the intensity gradient of the laser spot, responsible for trapping, and a scattering force, proportional to the light intensity that tends to push particles away from the laser focus destabilizing single-beam trapping of particles with large extinction. Such detrimental effects can be suppressed through the use of two counter-propagating beams to null the opposite scattering forces [13]. These dual-beam traps are based on the use of low numerical aperture (NA) lenses and allow the trapping of particles with reduced incident power in a focal region that is wider than for standard optical tweezers [10]. Thus they are well suited for operation in air or vacuum where detrimental effects by radiation pressure are enhanced by the reduced viscous damping [14-16].

Notwithstanding the remarkable improvement in optical trapping techniques, their application to planetary exploration (see the pictorial representation in Fig. 1) is still to be developed, even though already conceived by, e.g., NASA [17]. The development of the optical trapping technique to collect and analyze in situ or return to Earth a variety of extraterrestrial particles will open doors to information on space materials that are currently unreachable: (1) as the dust volatile component, not measurable in situ by dust instruments, e.g., those onboard Rosetta/ESA, and not retrievable by samples return missions, e.g., Stardust/NASA, Hayabusa; (2) have biases due to collection media contamination, e.g., aerogel used by the Stardust/NASA space probe $[18,19]$.

Here, we first review the role of dust in the universe: from interstellar to interplanetary, cometary, and planetary dust particles. Thus, we describe the models and methods we use to calculate light pressure and optical trapping properties on a variety of realistic dust particle models. Then, we show results on solar radiation pressure calculations that can help to a greater understanding of micro-particle dynamics as well as to estimate its detrimental role in optical trapping in space. Finally, we consider and compare results of calculations for optical trapping of dust particles in standard optical tweezers in water (typical laboratory conditions) with those calculated for OT in air or in space.

\section{A dusty universe}

We live in a dusty galaxy, one of the billions of galaxies in the universe. Almost all of them are dusty. Up to the mid-twentieth century, all of this dust was considered as an unfortunate impediment to making precise observations of stars and galaxies, regarded as the most important items in the universe. The modern view of dust as a cosmic component is almost entirely the reverse of that earlier view. We now know that almost every aspect of the formation of planets, stars, and galaxies is influenced in some way by interstellar dust [20]. The involvement of dust grains in providing molecules important for the origin of life and in the safe transmission of those species to newly forming planets orbiting Sun-like stars [21], has 


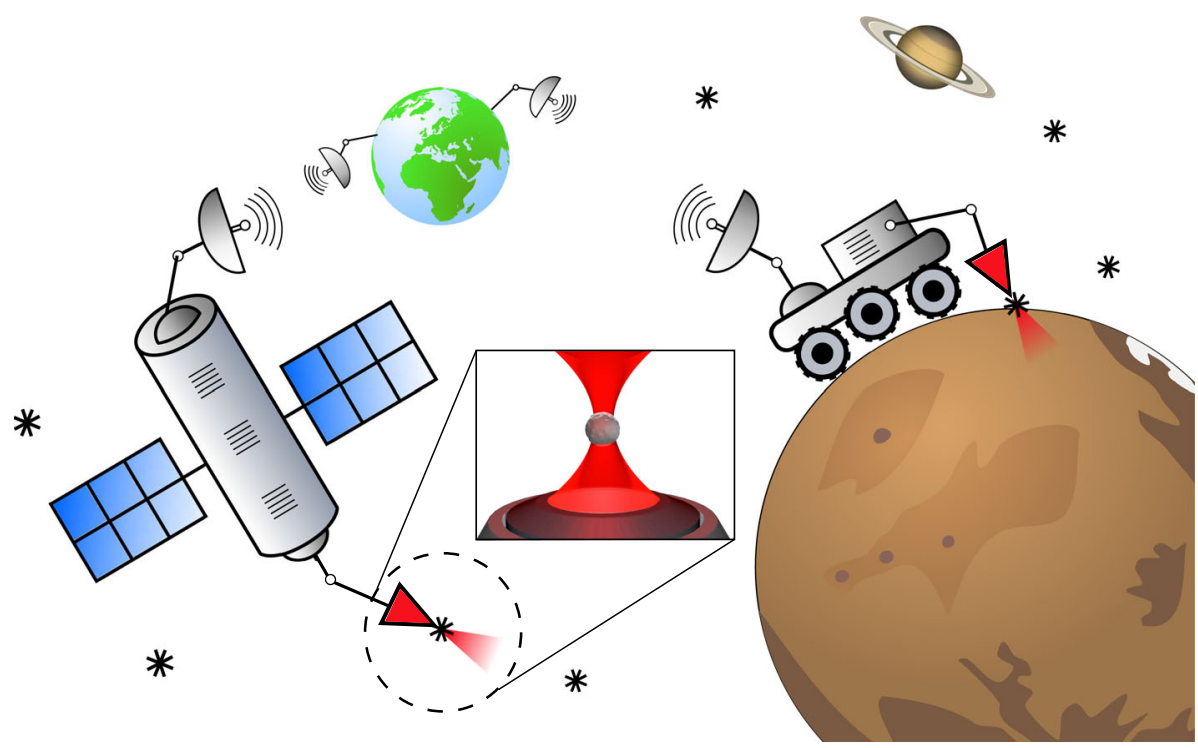

Fig. 1 Pictorial representation of space tweezers, space applications of optical tweezers. Interplanetary or planetary dust can be collected and investigated directly in situ (open space or extraterrestrial surfaces). The inset represents a closeup of a grain of interplanetary dust trapped by a single-beam optical tweezers.

been one of the greatest surprises of all. It is no wonder that considerable efforts are currently placed in trying to understand nature, composition, and evolution of dust in the interstellar medium of galaxies.

There is now a robust evidence that dust grains condense in circumstellar environments, mainly AGB (Asymptotic Giant Branch) stars [22] and supernovae [23,24], from which they are ejected in the general interstellar medium. There, dust is found to be tightly mixed with the gas, with the dust representing only a minor fraction of the total mass. The gas-to-dust mass ratio locally assumes the fairly constant value of 100 [25]. The dependence of this ratio on the metal content among galaxies and within a galaxy is an important issue from a cosmic perspective for a number of reasons [26], including the appearance of the first solids in the early universe [27]. In late-type galaxies, like our own, this ratio scales with the metallicity, decreasing with the galactocentric radius, a clear indication that dust growth in the interstellar medium dominates over destruction [28]. As a consequence, stardust, literally dust formed around stars, is not the same as interstellar dust and must be regarded as the raw material from which true interstellar grains are formed. Such a material is modified and destructured by violent interstellar processes [29], before being reformed and reassembled in denser interstellar regions [30].

Modifying processes continue to act throughout the lifetime of a dust grain, some hundreds of millions of years in the Milky Way galaxy. In some cases, such modifications may be catastrophic, as during the formation of a planetary system, a filter that severely modifies the composition of interstellar dust. Those materials passing through the filter are the most robust to destruction and erosion. In the solar system, the patterns of isotopic abundances that are found in survived presolar grains clearly identify their origins in the cool envelopes of evolved low mass stars and in supernovae ejecta. These particles have passed through many episodes of possible destruction, including their ejection from the stellar envelopes, their passage through the interstellar medium, where they have been subjected to intense radiation 
(a)

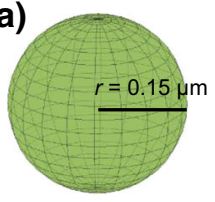

(e)

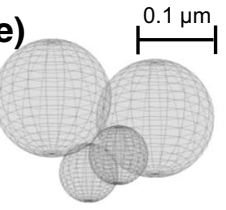

(i)

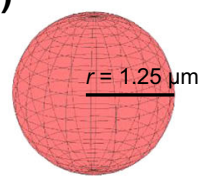

(b)
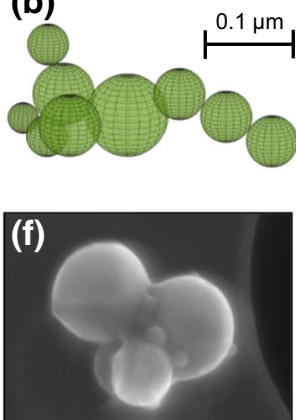

(j)

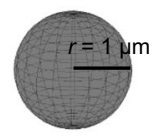

(c)
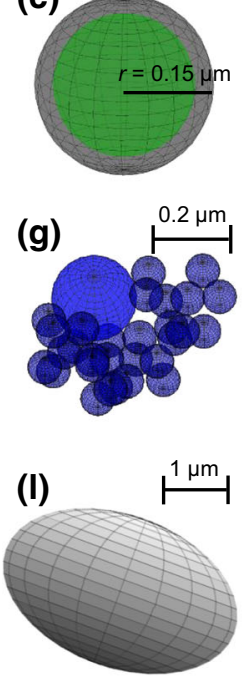

(d)
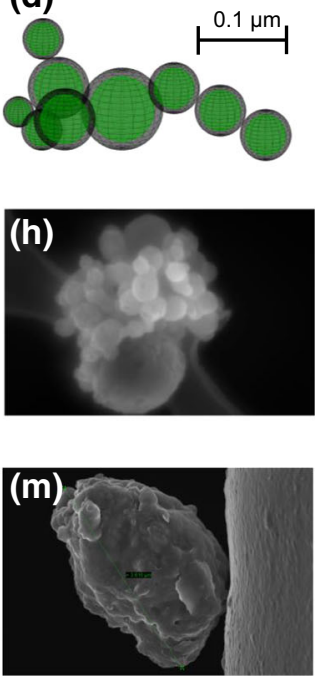

Fig. 2 Scattering models with shape and composition inspired by interstellar, interplanetary (DUSTER mission [31]), and planetary [32] particles. The models emulate hypothetical interstellar dust grains whose constituents are olivine and aliphatic carbon. In $\mathbf{a}$ and $\mathbf{b}$, the constituents refractive indexes are mixed according to the Bruggeman criterion. Instead, in $\mathbf{c}$ and $\mathbf{d}$, the olivine is considered covered by a carbon layer. In $\mathbf{e}$, the model of a silica particle arranged in quenched melt spheres shown in the FESEM image [31] f. In $\mathbf{g}$, the model of condensed $\mathrm{Ca}[\mathrm{O}]$ nanograins that are accreted onto a larger melted aggregate of tiny carbonate grains shown in the FESEM image [31] (h). The larger sphere is calcite and the other spheres are $\mathrm{CaO}$. In i, a spherical model of the particle Fe, Mg-rich 'TP2' [19], in which we consider an effective refractive index constructed mixing iron (67\%) and magnesium (33\%), according to the Bruggeman criterion. In $\mathbf{j}$, a spherical model of Martian hematite [32]. In k, a spherical model of Lunar regolith [33]. In l, a model of an ellipsoidal fassaite shown in the FESEM image $(\mathbf{m})$. Here, we consider an effective refractive index constructed mixing silica (53\%), $\mathrm{CaO}(27 \%), \mathrm{FeO}(10 \%), \mathrm{Al}_{2} \mathrm{O}_{3}$ (10\%), according to the Bruggeman criterion [34]

fields and dynamical shocks, their incorporation into the molecular cloud that formed the solar system, and through all the varied and violent processes involved in the formation of the Sun and its planets. As near-stellar dust is modified in the interstellar medium and becomes interstellar dust, interstellar dust may be severely modified, when not obliterated, when it is incorporated in the gas that forms newly born planetary system.

\subsection{Interstellar dust}

Most of the information that we have about interstellar dust is obtained remotely, by the influence of dust on various kinds of astronomical observations. These observations may be carried out at a very wide range of wavelengths, from X-rays to radio waves, but traditionally the most important and defining data have come from the infrared, visible, and ultraviolet parts of the spectrum, summarized through a wavelength-dependent extinction curve along the lines of sight to individual stars.

There are detailed extinction measurements along hundreds of lines of sight in the Milky Way Galaxy [35] and less accurate extinction data for the interstellar media of external galaxies [36]. Such observed extinction profiles are different, but are clearly members of the same family showing a basic similarity in their shapes: The extinction typically increases from low values in the infrared to high values in the far ultraviolet, a near-linear portion in 
the optical region, (in most cases) a pronounced and broad "bump" near $217.5 \mathrm{~nm}$, and a final rise of varying slope into the far ultraviolet. Both the general aspect of the extinction and the details of specific curves along particular lines of sight provide useful information and generally indicate that grains of a wide range of sizes (roughly nanometers to micrometers) are required [37].

The dominant feature in the extinction curve is the prominent bump in the near ultraviolet. Its central position is fixed, although the width of the feature can vary significantly from one line of sight to another. It is widely attributed to $\pi^{\star} \leftarrow \pi$ transitions in aromatic carbon solids [38] or polycyclic aromatic hydrocarbons [39]. The far-ultraviolet rise may be decomposed in a linear contribution, due to nano-sized particles, and a nonlinear component belonging to the partially invisible (because located beyond the Lyman continuum) $\sigma^{\star} \leftarrow \sigma$ resonance in aromatic carbon. In the near-infrared, there is a weak absorption feature at a wavelength of $3.4 \mu \mathrm{m}$, detectable on long paths through diffuse gas. It is characteristic of absorption in the $s p^{3}$ (aliphatic) $\mathrm{C}-\mathrm{H}$ stretching bond [40]. Further into the infrared are two stronger absorption features, at 9.7 and $18 \mu \mathrm{m}$, ascribable to silicate materials, from $\mathrm{Si}-\mathrm{O}$ stretching and $\mathrm{O}-\mathrm{Si}-\mathrm{O}$ bending modes, respectively. Taken together, these features strongly support the presence of some form of carbon/hydrocarbon and silicate in the dust. X-ray scattering and absorption edges provide constraints on grain size and composition, specifically $\mathrm{O}, \mathrm{Mg}, \mathrm{Si}$, $\mathrm{Fe}$, and $\mathrm{C}$ atoms [41]. There is also a set of detected infrared emission features occurring at $3.3,6.2,7.7,8.6$, and $11.3 \mu \mathrm{m}$, indicative of aromatic $\mathrm{CH}$ groups. The mechanism responsible for the excitation of such infrared emission may involve non-equilibrium emission from polycyclic aromatic hydrocarbons stochastically heated to high temperatures by the absorption of individual photons from the interstellar radiation field [42]. However, the requirement on microscopic sizes can be relaxed if the emitters of the $3.3 \mu \mathrm{m}$ and other infrared bands are heated by the chemical energy released from reactions within larger carbon interstellar grains of mixed $s p^{2} / s p^{3}$ carbon composition [43]. Such structures have been in fact observed in some extragalactic objects [44]. Exploiting a ternary phase diagram where the hydrogen content and the two main bonding types $\left(s p^{2}\right.$ and $\left.s p^{3}\right)$ for carbon constitute the poles, Dartois et al. [44] were able to identify the carrier of the spectral features as an interstellar hydrocarbon belonging to the class of polymeric-like hydrogenated amorphous carbon $(\mathrm{a}-\mathrm{C}: \mathrm{H})$, dominated by an aliphatic/olefinic backbone structure. The change from aliphatic to aromatic structures may occur in environments that selectively dehydrogenate the a-C:H, providing an opportunity for aromatic molecules to form. These observations, together with observations of very evolved stars (protoplanetary and planetary nebulae), suggest an evolution in which aliphatics are converted into aromatic structures [45,46].

The interpretation of dust observations must take also account of the available abundances along the line of sight. The hydrogen abundance is often well-determined; abundances of other elements relative to hydrogen are assumed, using solar or other relative values as a standard. It is unclear which of these standards is the appropriate one to use [47], but whichever one is adopted, the inventory of some materials is demonstrably incomplete. In fact, silicates alone are unable to account for the entirety of either the oxygen solid phase or Fe abundances [48]. The remainder of the Fe could be in iron oxides or in metallic form [49]. The unaccounted oxygen is less readily explained [50]. There is an evident problem of oxygen budget in the dense interstellar medium, as the combined contribution of gaseous $\mathrm{CO}$ and silicate/oxide dust is by far less of the lowest reference abundances [51]. Solid $\mathrm{H}_{2} \mathrm{O}$ is an obvious O-bearing candidate. Astronomers have known for decades that water is fairly common in the universe. Interstellar water ice, as distinct from gaseous water molecules, was first identified toward the embedded protostellar Becklin-Neugebauer object by Gillette and Forrest [52]. It is now widely detected in interstellar dark clouds. These ices, composed 
mainly of water, also contain carbon monoxide and dioxide, simple hydrides such as methane and ammonia, and a few other species [53]. Still, ice contribution is not enough. According to Whittet [50], the most plausible candidate for the depleted oxygen appears to be a form of O-bearing carbonaceous matter similar to the organics found in cometary particles returned by the Stardust mission. Such materials may share some similarities with refractory organic residues arising in the irradiation of icy mixtures by ions [54] or X-rays [55].

For its very nature, astronomical dust is likely to be an amalgam of a number of different materials, very chaotic in composition and structure, with different individual substances dominating at different wavelengths. These materials are thus fundamentally different from terrestrial materials. Nuth and Hecht [56] introduced the concept of chaotic silicates in which the level of disorder is even greater than for glasses, that are characterized by the absence of long-range order in the atomic arrangement beyond nearest neighbors. Since materials may be assembled in the agglomerate, an astronomical silicate cannot be considered a solid with a definite stoichiometric composition. They may also occur in groups that recall solid solutions, in which one or more types of atoms or molecules of the solid may be partly substituted for the original atoms and molecules without changing the structure. Olivine and enstatite are excellent examples of solid solutions. Forsterite, $\mathrm{Mg}_{2} \mathrm{SiO}_{4}$, and fayalite, $\mathrm{Fe}_{2} \mathrm{SiO}_{4}$, have identical structures because the ions $\mathrm{Mg}^{2+}$ and $\mathrm{Fe}^{2+}$ are very nearly the same size and are chemically similar. Very frequently amorphous silicates in space are misleadingly described in terms of the optical properties of these materials. Indeed, as pointed out by Rietmeijer and Nuth [57], there are no amorphous silicates, as the word "silicate" already implies that the material is crystalline and could be a mineral. Moreover, astronomical solids may be porous and therefore of much lower density than a glass. Ultimately, the nature of an astronomical silicate is rather loosely constrained, to same extent just limited to a material whose infrared spectrum is dominated by $\mathrm{Si}-\mathrm{O}$ stretching and bending vibrations. Thermal annealing (e.g., in shocks) or intense X-ray irradiation [58] of precursor materials, that were probably amorphous, may explain the presence of crystalline silicates (see however Ritmejer and Nuth 2013 [57], for petrologic constraints) in circumstellar regions and protoplanetary disks [59].

Even carbonaceous materials in space are difficult to constrain. A striking example is given by the nature of the carrier of the interstellar ultraviolet extinction bump at $217.5 \mathrm{~nm}$, that was originally attributed to small crystalline graphite particles [60], followed by a plethora of proposals including mixture of spheres composed of graphite, amorphous carbon, and silicate [61], irregular or fractal arrangement of graphite and amorphous carbon [62], polycyclic aromatic hydrocarbons [63], natural coal [64], and even electronic transitions of $\mathrm{OH}^{-}$ions in sites of low coordination in silicates [65]. In general, carbonaceous materials contain greater or lesser hydrogen fractions, varying proportions of different chemical bonding, and different degrees of long-range order. All these forms of carbon can, under suitable conditions, be readily converted from one to another. The manifold of possible bonding arrangements produces several allotropes of carbon of which the best known are graphite, diamond, and amorphous carbon. The physical properties of carbon vary widely with the allotropic form. Amorphous carbonaceous materials cover a wide range of compositions, from wide band gap, H-rich, aliphatic-rich a-C:H to narrow band gap, H-poor, aromatic-rich a-C materials. The properties of a-C:H materials are determined by the $s p^{3} / s p^{2}$ ratio for the carbon atoms and the hydrogen concentration. A $\mathrm{C}-\mathrm{H}$ bond contributes to the formation of $s p^{3}$ bonding and the reduction of the defects in the amorphous carbon network. In general, it is found that the optical energy gap increases with hydrogen concentration [66].

Remote observations such as extinction profiles were at one time the only sources of information about interstellar dust. However, even if challenging it is now also possible to 

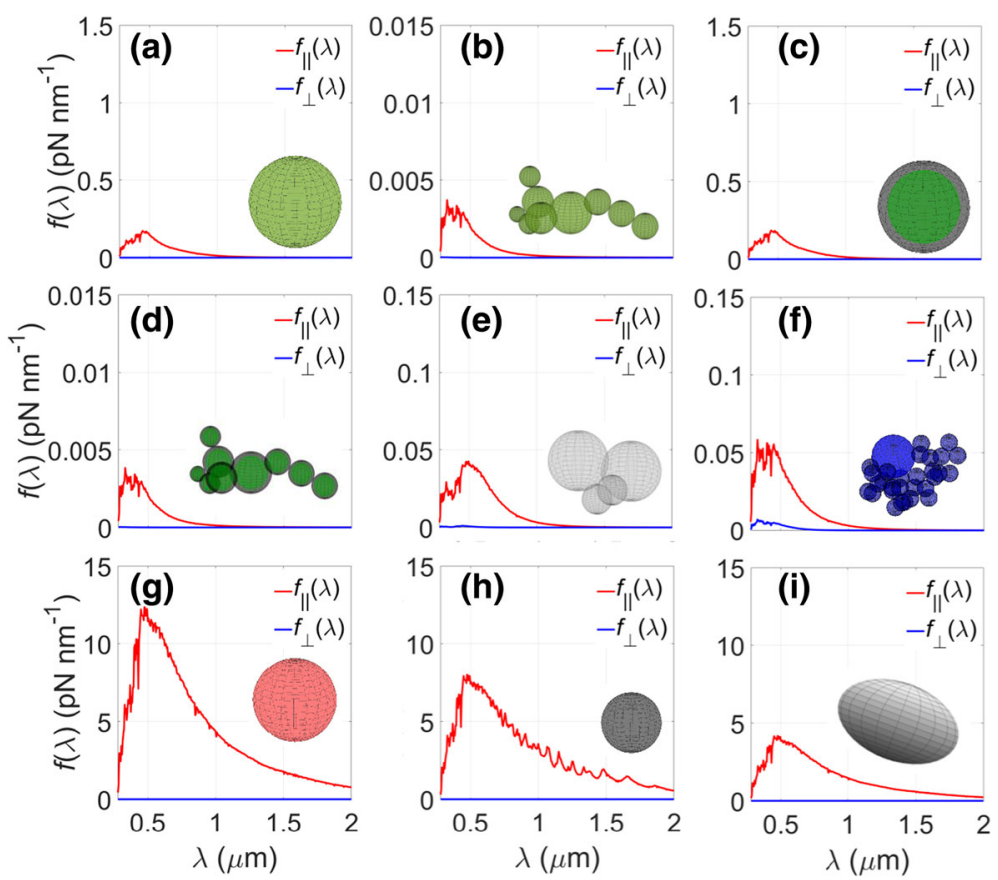

Fig. 3 Optical forces distributions exerted by the Sun on dust particles modeled as shown in Fig. 2. The optical force parallel, $f_{\|}(\lambda)$ (blue line), and perpendicular, $f_{\perp}(\lambda)$ (red line), are intended respect to the light propagation direction $\hat{\mathbf{k}}$. In a-d, we show calculations on olivine-aliphatic carbon structures. In $\mathbf{a}$ and $\mathbf{b}$, the constituents refractive indexes are mixed according to the Bruggeman criterion [34]. In $\mathbf{c}$ and $\mathbf{d}$, the olivine and the carbon are distributed in a core-shell structure. In $\mathbf{e}$, we show results for the quenched melt silica particle, in $\mathbf{f}$ for the bunch-of-grape carbon Ca-rich, in $\mathbf{g}$ for the 'TP2' sphere, in $\mathbf{h}$ for the martian hematite sphere, and in $\mathbf{i}$ for the fassaite ellipsoid

study in situ interstellar grains entering the heliosphere by spacecrafts and to collect them using stratospheric aircrafts and balloons and satellite probes. Rare interstellar dust grains can now be examined in the laboratory being returned by the Stardust/NASA mission. During the Cassini mission around Saturn, millions of dust particles have been analyzed, and now, thanks to the Cosmic Dust Analyzer on board the probe, 36 grains of dust supposed coming from outside our solar system have been detected [67]. Results from different missions generally deviate one from another, and typically they are not very consistent with the dust picture obtained by remote observations [30]. Obviously, this is a field in which much work is required.

\subsection{The interplanetary dust complex}

The interplanetary space of the solar system is very dusty, appreciable to the naked eye through the faint solar color cone of light above the western horizon after sunset or above the eastern horizon just before sunrise: the Zodiacal Light, a huge amount of fine dust particles that scatter solar radiation in the interplanetary space. The brightness of the Zodiacal Light provides information on the overall space density of the dust known as the interplanetary dust complex. 
The interplanetary dust complex consists of microscopic (mainly rocky) particles, typically less than a few millimetres in size, called micrometeoroids, moving in the interplanetary space of the solar system [68]. Dust is produced by collisions among solid bodies, by disruptions of icy bodies [69,70] and by cometary activity [71,72]. As such the interplanetary dust complex is an inventory of the constituent materials of a large variety of solid bodies in the solar system. Their cosmochemical study thus allows investigation of great diversity of astrogeobiological processes occurred on their parent bodies. Remarkably, these include the most primitive bodies not further processed by planetesimal accretion after aggregation in the protoplanetary disk [73]. In addition, since the bulk of extraterrestrial matter accreted by the Earth is in the form of cosmic dust from the interplanetary dust complex, understanding its composition provides also clues on outstanding issues like the contribution of extraterrestrial matter to the Earth's geochemical budget [74], including its bearing on the emergence of life [75]. Based on particle-impact detection at the Long Duration Exposure Facility/NASA, the main mass fraction of the submillimetre dust particles in the Zodiacal cloud in the vicinity of the Earth have characteristic diameters of 100 to $200 \mu \mathrm{m}$, and the flux of micrometeoroids entering the Earth's atmosphere is 40,000 $\pm 20,000$ tonnes per year [76]. Grün et al. [77] reached a similar conclusion and quantified the spatial mass of cosmic debris at $1 \mathrm{AU}$ as $10^{-16} \mathrm{~g} \mathrm{~m}^{-3}$, with the largest fraction in the $10^{-6}$ to $10^{-4} \mathrm{~g}$ mass range, based on the lunar microcrater record and spacecraft micrometeoroid detectors.

How much asteroid collisions versus cometary activity contribute to the interplanetary dust complex is an outstanding issue. Matter is complicated since such contributions are expected to vary through the solar system's history; this is due to the stochastic fluctuations in the number of mutual collisions in the asteroid belt and the number and level of activity of comets entering the inner solar system. If astronomical observations carried out by the Infrared Astronomical Satellite (IRAS) and Cosmic Background Explorer (COBE) satellites in the 1980s indicated that collisions in the main asteroid belt (MAB) are the dominant source of dust in the near-Earth space [78], dynamical simulations predict in turn that the bulk of the Zodiacal cloud can be best produced by debris derived from Jupiter family comets (JFCs) through spontaneous disruption [79]. Oxygen isotopic data from relatively large micrometeorites in the 100-1000 $\mu \mathrm{m}$ size range collected at the Earth's surface [80-82] indicates that the interplanetary dust complex is dominated by dust produced by cometary activity and by collisions between primitive hydrous asteroids of carbonaceous chondrite compositions, with a subordinate contribution from more evolved anhydrous asteroids (mainly ordinary chondritic and Vesta-like). An additional small fraction of micrometeorites, with heavy oxygen isotope composition, may sample an unknown body in the solar system, namely a body not yet sampled by macroscopic meteorites. The isotopic statistics shows that primitive, volatile-rich material dominates the small size fraction, whereas evolved anhydrous material dominates the large size fraction of the dust complex.

Physical and compositional properties of the interplanetary dust complex Most of the compositional properties of the interplanetary dust complex derives from the cosmochemical analyses of samples recovered from: (1) Earth's surface, e.g., micrometeorites collected in Antarctica [80,81,83]; (2) Earth's stratosphere, by balloon born instruments, e.g., DUSTER, designed for nondestructive and uncontaminated collection of solid particles from tens of microns down to $200 \mathrm{~nm}$ in size [84] and by stratospheric NASA/aircraft passive sticking on silicon oil-coated plates [85]. A critical contribution is also given by laboratory analyses of samples, i.e., collected, and brought back to Earth, from asteroid surfaces [86] and in a cometary coma $[18,19]$. In addition, cometary dust were studied in situ from the onset of cometary activity to its cessation after perihelion by the Rosetta/ESA space mission [87-89]. 
The pristine mineralogy of micrometeorites is best unraveled by the study of a specific class: the unmelted, i.e., particles that did not experienced alteration by frictional heating during atmospheric passage [90]. Based on petrographic and geochemical data, most of the micrometeorite flux reaching Earth today is dominated ( $>50 \%$ of unmelted micrometeorites across all size fractions) by fine-grained and hydrated carbonaceous chondrite material affine to the CM, CR matrices and CI chondrites, with some specimens similar to the ungrouped meteorite Tagish Lake. They are therefore a major component of the near-Earth dust complex in agreement with isotopic statistics [80]. Coarse-grained micrometeorites, which are primarily fragments of chondrule, and $\mathrm{CAI}$ (Ca- and Al-rich inclusions) represents a smaller component $(<25 \%)$ of the micrometeorite flux [81,91]. Thus, the major constituent minerals are olivine, low-Ca pyroxene, magnetite, sulfides, metal, and hydrous $\mathrm{Fe}-\mathrm{Mg}$ silicates like serpentine and saponite. The density of unmelted micrometeorites varies greatly according to their mineral composition. Mean density for unmelted fine-grained micrometeorites is $\sim 1.4 \mathrm{~g} \mathrm{~cm}^{-3}$ (average data from Kohout et al. [92]), but can be more than twice for coarse-grained micrometeorites. Micrometeorites range in size from 10 to $2000 \mu \mathrm{m}$ [68]; however, particles up to $3000 \mu \mathrm{m}$ in size have been reported in the Transantarctic Mountains micrometeorite collection [93,94], and their mass varies within the nanogram to the milligram range. A statistically significant number of micrometeorites in the $200-700 \mu \mathrm{m}$ size range shows that the micrometeorite size distribution is bimodal, with peaks centered at $\sim 145$ and $\sim 250 \mu \mathrm{m}$. This suggests that the micrometeorite flux is composed of multiple dust sources with distinct size distributions: fine-grained material from primitive objects and coarse-grained material from evolved bodies [81].

DUSTER particles, collected with a strict contamination protocol during different stratospheric balloon flight campaigns at 30-40 km altitudes, are the smallest (down to $200 \mathrm{~nm}$ ) meteoritic objects available for laboratory investigation (see Fig. 2f, h, l). These are the residues of porous, structurally weak bolides crossing the Earth's atmosphere. They contribute to the daily input of stratospheric extraterrestrial material with: (1) tens of microns down to sub-micrometre aggregates of nanometer-sized spherical grains, produced by vaporization and quenching; (2) partially annealed minerals; and (3) unprocessed minerals [31,95]. DUSTER particles composition includes alumina, aluminosilica, plagioclase, fassaite, silica, $\mathrm{CaCO}_{3}, \mathrm{CaO}$, extreme F-rich $\mathrm{C}-\mathrm{O}-\mathrm{Ca}$ particles, and oxocarbon. These are particle linked to the friable $\mathrm{CI}$ and $\mathrm{CM}$ carbonaceous chondrite and unequilibrated ordinary chondrite meteors that are the most common source of bolides and fireballs [31].

Similar particles are collected at lower quotes in the stratosphere by NASA/aircrafts: interplanetary dust particles (IDPs), numerous and fine-grained, are available for laboratory analyses $[85,96]$. They have a chondritic overall composition, masses in the order of picoto nano-grams and sizes of few microns [68], but they can reach hundreds of microns for the giant cluster IDPs class. They mainly consist of extremely fragile aggregate of crystalline and amorphous materials with grain size typically in the order of $100 \mathrm{~s}$ of $\mathrm{nm}$ or smaller. Hydrous IDPs are mainly massive objects with fibrous or platy surface textures. They consist mainly of hydrous mineral assemblages, mostly smectite and lesser serpentine and silicate glass, with minor amounts of diopside, forsterite, chromite, Fe- and Ni-sulfides, schreibersite (Ni-phosphide), magnetite, glasses of silicatic composition and disordered carbonaceous material. Among IDPs are the aggregate anhydrous IDPs, which are extremely fine-grained, highly porous (up to $70 \%$ ) and low-density $\left(0.3-0.6 \mathrm{~g} \mathrm{~cm}^{-3}\right)$. Following Rietmeijer's classification[85], they consist of a matrix aggregates of generally spherical entities, $\sim 0.1$ to $\sim 1$ micron in size, with embedded variable amounts of $\sim 5$ micron-sized $\mathrm{Mg}$, Fe silicates and $\mathrm{Ca}, \mathrm{Mg}$, Fe silicates, Ni-free and low-Ni pyrrhotite, iron oxides, and amorphous materials. Aggregate IDPs are supposed to have a cometary origin [97] testified also 
by the comparison with cometary dust particles returned by the Stardust/NASA space probe $[18,19]$. In fact, dust particles after ejection from the nucleus form a dust flux [98], which undergoes to solar radiation pressure effects, as observed in situ by GIADA onboard the ESA Rosetta space probe [99], to gas drag and to gravity force [100]. These dust particles either remain tied to the nucleus or they are liberated into space feeding the interplanetary dust complex, where their orbital evolution is mainly controlled by radiation pressure and the Poynting-Robertson light drag [101]. This causes particles from 1 to $1000 \mathrm{~s}$ of $\mu \mathrm{m}$ in size to spiral slowly into the Sun, i.e., to gradually reduce the orbit's eccentricity and semimajor axis, in a $10^{4}$ to $10^{6}$ year time scales, intercepting in some cases Earth's atmosphere, where they are collected as IDPs.

\subsection{Planetary dust}

Many terrestrial planets and satellites of both terrestrial and giant planets in our solar system show dusty environmental conditions. In particular, the mostly explored dusty bodies are the Moon and Mars.

The Moon. The Moon is the only Earth's natural satellite. Since the Moon has neither a magnetic field nor a significant atmosphere, the lunar regolith and the near-surface environment are mainly affected by space weathering processes such as meteoroid impacts, solar ultraviolet radiation, solar wind, galactic cosmic rays, and plasma processes in the tail of the Earth's magnetosphere.

On the Moon, all the locations explored so far have cratered surfaces covered with loose regolith of several meters. We can therefore assume that the entire surface is covered by regolith although the thickness may vary (about $5 \mathrm{~m}$ in the maria, up to $20 \mathrm{~m}$ in the highlands). Such regolith layer is a cohesive, dark gray to light gray, very fine-grained, clastic material consisting of a mixture of a wide variety of materials including fragments of highland anorthosites, some forms of KREEP volcanic rocks, mare basalts, and volcanic glass, plus a small meteoritic component [102]. The influence of the solar wind and high-energy particles of solar and cosmic origins induces the implantation of $\mathrm{H}, \mathrm{He}$, and many rare elements into the regolith. Continued reworking by micrometeoroids of the hydrogen-enriched regolith particles causes the material to melt and react (particularly, $\mathrm{H}$ with $\mathrm{FeO}$ ), producing water vapor and submicron grains of metallic iron sintered into glass [103].

Considering the samples returned by the Apollo and Luna missions, the fine fraction of the lunar regolith (45-100 $\mu \mathrm{m}$ in mean size) comprises about a half of the lunar regolith by weight; the superfine fraction (particles smaller than $10 \mu \mathrm{m}$ ) about $10 \%$, while the particles smaller than $2 \mu \mathrm{m}$, the finest regolith fraction, make up 1-2\% of the mass. The shape of dust particles is extremely irregular and highly variable, ranging from angular with sharp edges to spheroidal. The density of individual particles is usually assumed to be in the range from 2.7 to $3.0 \mathrm{~g} \mathrm{~cm}^{-3}$ [104]. Individual particles may be glass-bounded aggregates [103] called agglutinates. Agglutinate particles may have lower density (higher porosity), they are usually $<1 \mathrm{~mm}$ and contain minute droplets of Fe metal (much of which is very fine-grained, single domain $\mathrm{FeO}$ ), and troilite (FeS). They formed by the melting and mixing produced by micrometeoritic bombardment of the lunar regolith.

The optical parameters of the dust particles (i.e., the real and imaginary parts of the complex refractive index) depend on the composition and can vary significantly, not only over a wide region, but even locally in a microscopic scale. For the glassy component, the real part values range from 1.570 to 1.749 , and they vary directly with the total $\mathrm{Fe}$ and $\mathrm{Ti}$ contents and inversely with the Al content [105]. The imaginary part of the complex refractive 
index is more variable and can span from 0.0005 up to 0.15 according to the composition and wavelength of interest.

Electrical properties of the dust particles are characterized by the extremely low electrical conductivity (approximately $10^{-14} \mathrm{ohm} / \mathrm{m}$ in shadowed areas [104], but about a hundred times higher under solar light exposure) permitting accumulation of electrostatic charge under ultraviolet irradiation.

Mars. Mars, the fourth planet of our solar system, has many features in common with the Earth. Much of the Martian surface is covered by unconsolidated soils (dust) derived from impact, eolian and other sedimentary processes. Such dust is likely produced by impacts early in Mars' history and subsequently recycled at its surface. Sometimes the wind in Mars' thin atmosphere blows the dust on the surface into dust storms, carrying dust particles up to altitudes of about $50 \mathrm{~km}$. The composition of the Martian dust grains was obtained from several space missions, starting from the mission Mariner 9 in 1971 [106].

It is possible to build a mineralogical model of the dust using all the data acquired from the Mariner 9 and Viking Landers [107] space missions. Best fit results are those obtained by mixing different kinds of clay, with montmorillonite $\mathrm{Si}_{2} \mathrm{Al}_{4} \mathrm{O}_{10}(\mathrm{OH})_{2} \cdot \mathrm{nH}_{2} \mathrm{O}$ as a base, mixed with nontronite (where part of $\mathrm{Al}$ is replaced by $\mathrm{Fe}^{3+}$ ) and saponite (where $\mathrm{Al}$ is replaced by $\mathrm{Mg}$ ). Indeed, data by the $\mathrm{X}$ spectrometer aboard the Pathfinder showed higher abundances of magnesium and aluminum and lower abundances of iron, chlorine, and sulfur [108] respect to what expected. The rocks analyzed were similar to andesites, terrestrial materials derived from the fractionation of basalt in intrusive conditions.

Data from recent rovers (e.g., MSL Curiosity at Rocknest, Oct 2012) and orbital spacecraft show that Martian surface is dominated by a soil (dust grain dimension $<150 \mu \mathrm{m}$ ) of basaltic composition with primarily pyroxene, plagioclase feldspar, and olivine, as well as minor amounts of $\mathrm{Fe}$ and $\mathrm{Ti}$ oxides (e.g., magnetite, ilmenite, and hematite) and alteration minerals (e.g., sulfates, phyllosilicates, and carbonates) [109-112]. Dust particles properties changes during dust storms [113]. In general, dust particle sizes range from 1.2 to about $4.1 \mu \mathrm{m}$ with a mean dust radius of about 1.6-1.8 $\mu \mathrm{m}[114,115]$ and a positive correlation between dust opacity and particle sizes.

Phase function results show asymmetry parameter values of $g=0.601 \pm 0.108$ for high atmospheric dust loading scenarios and $g=0.710 \pm 0.065$ for non-dusty periods. Regarding the shape of the particles, considering a modified log-normal aspect ratio distribution for a mixture of spheroids, data suggest more elongated particles are present during dust storms, with aspect ratios of $2.8 \pm 0.9$ for high-opacity days, in contrast to values of 1.8 measured during post-storm period. The particles single scattering albedo is found ranging in the solar band between 0.89-0.90 (dark regions) and 0.92-0.94 (bright regions) [116].

Moreover, there are a very strong and a fast feedback between sand/dust emission and electric field enhancement and a linear relation between the concentration of lifted dust and the generated electric field [117].

\section{Methods and models for space tweezers calculations}

Optical forces and optical trapping are the consequence of the electromagnetic momentum conservation during a light scattering process $[5,118]$. To understand theoretically how light interacts mechanically with matter, Maxwell's equations and the integration of the averaged Maxwell stress tensor have to be performed [118,119]. However, such calculations can be computationally complex, e.g., when we deal with non-spherical or non-homogeneous particles, and the use of approximations can be also advantageous $[5,120]$. The range of 

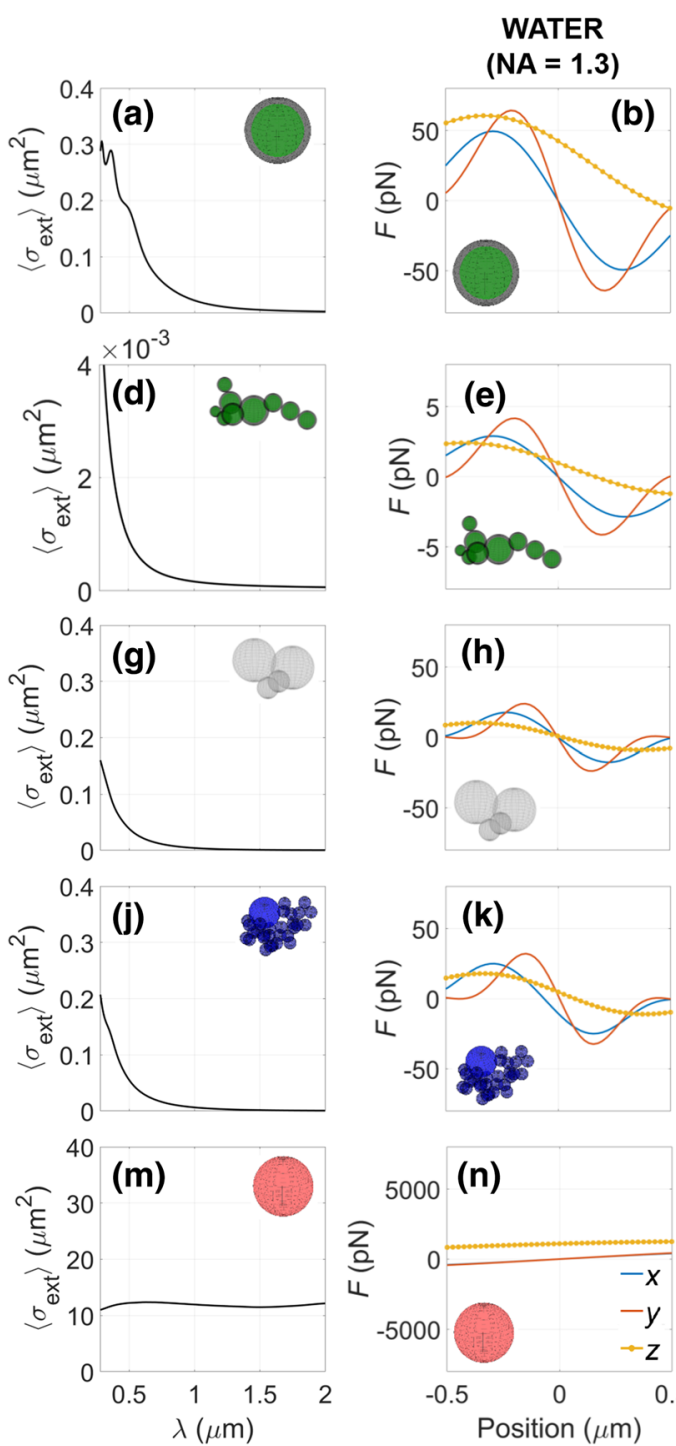
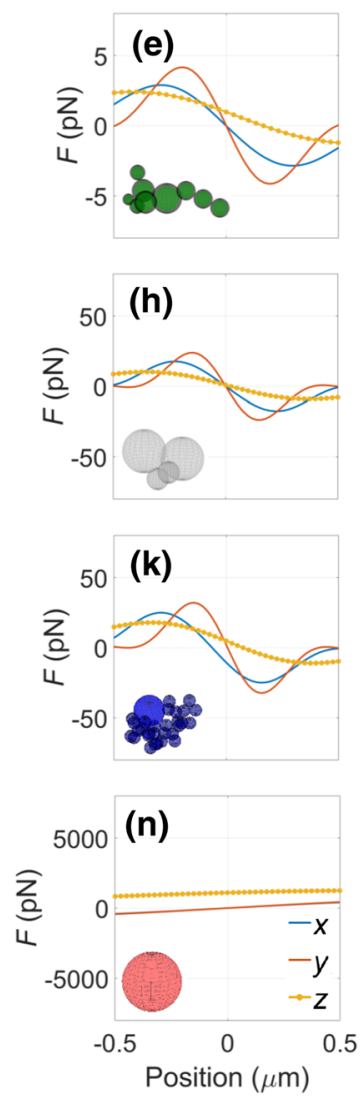

AIR/VACUUM

(NA $=0.9)$
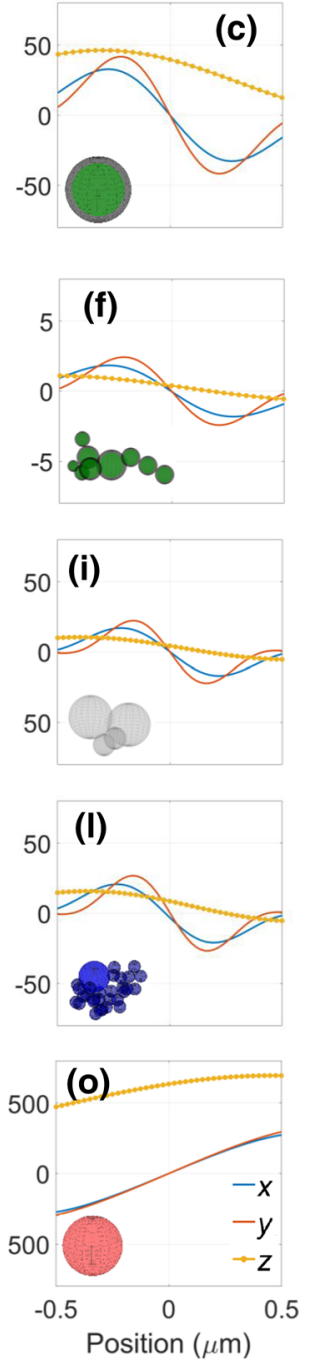

Fig. 4 Extinction cross sections, $\left\langle\sigma_{\text {ext }}\right\rangle$, and optical trapping force, $F$, components along $x$ (blue line), $y$ (red line), and $z$ (yellow dots) when the particle is trapped in water (center column) or in air (right column). Results in $\mathbf{a}-\mathbf{c}$ are related to the olivine-aliphatic carbon core-shell sphere. $\mathbf{d}-\mathbf{f}$ are calculations for the olivine-aliphatic carbon core-shell cluster. $\mathbf{g}-\mathbf{i}$ are the quenched melt silica. $\mathbf{j}-\mathbf{l}$ are the results for the bunch-of-grape carbon Ca-rich. $\mathbf{m}-\mathbf{o}$ are the results for the 'TP2' sphere. For the optical trapping calculations, the laser power is fixed at $50 \mathrm{~mW}$ and the wavelength at $0.83 \mu \mathrm{m}$

validity of the different approximations is determined by the size parameter $x=k_{\mathrm{m}} r$, where $k_{\mathrm{m}}=2 \pi n_{\mathrm{m}} / \lambda_{0}$ is the radiation wave number in the medium surrounding the particle, $r$ is the particle size, $\lambda_{0}$ is the wavelength in vacuum, and $n_{\mathrm{m}}$ is the medium refractive index [5]. When $x \ll 1$, the particle size is much smaller than the wavelength, and the dipole approximation can be adopted, treating the particle as a dipole [121-123]. In the opposite case, when $x \gg 1$, the ray optics regime appears computationally the most suitable, and the optical fields 
are simply represented as a collection of $N$ light rays according to the geometrical optics $[5,124]$. Each ray has a $N$-th portion of the total incident power $P_{\mathrm{i}}$, and a linear momentum per second $n_{\mathrm{m}} P_{\mathrm{i}, N} / c$ [125]. According to the Snell's law, a single ray which impinges on a surface with an certain incident angle is partly reflected and partly transmitted. Therefore, the ray changes its direction and, consequently, its momentum causing a reaction force on the center of mass of the particle [126].

In the intermediate regime, that is when the particle size is comparable with the light wavelength $(x \simeq 1)$ or for non-spherical and non-homogeneous particles like cosmic dust, we need to resort to full electromagnetic theory and the T-Matrix formalism has proved to be computationally advantageous $[118,127,128]$. Any particle can be modeled through a sphere, clusters or aggregates of spheres, spheres with spherical (eccentric) inclusions, and multilayered spheres [118]. This formalism consists in the expansion of the electromagnetic fields into a basis of vector spherical harmonics applying the boundary conditions across the particle surface $[118,119]$. The incident and the internal fields inside the particle have to be regular at the origin while the scattered field is such as to satisfy the radiation condition at infinity $[118,129,130]$. The transition matrix, $\mathbb{T}$, connects the amplitudes of the scattered fields to the amplitudes of the incident ones upon a multipole expansion of the fields. Its order is, in principle, infinite, and thus, it must be truncated to a multipole index value $L_{M}$ [118]. Such value is chosen to ensure the required accuracy of the transition matrix elements. However, for a cluster of $N$ spheres this implies the solution of a system of order $D_{M}=2 N L_{M}\left(L_{M}+2\right)$, which may become too large [118]. Actually, the inversion of the scattering matrix is responsible for most of the computation time required for the calculation that scales as $D_{M}^{3}$. Thus, on account of the definition of $D_{M}$, the computation time scales as $L_{M}^{6}$ whereas the storage requirements scale as $L_{M}^{4}$, so that it pays, in terms of both CPU time and storage requirements, to keep $L_{M}$ as low as practicable. Thus, the choice of an appropriate value of $L_{M}$, in order to satisfy computation time and storage requirements, is of crucial importance [131,132].

The diversity of dust particles in an astrophysical context implies a richness of models that we need to build in order to calculate realistic optical forces for space tweezers applications. Here, we consider several models of extraterrestrial dusts with shape and composition inspired by interstellar particles, DUSTER samples [31,95], and Moon or Mars dust analogs. We show these models in Fig. 2. On the top row, the homogeneous and stratified single/aggregated spheres emulate hypothetical interstellar dust grains whose constituents are olivine and aliphatic carbon [45]. The olivine refractive index is provided by Draine and Li while the carbon one by Ashok et al. [133,134]. In Fig. 2a and b, the constituents refractive indexes are mixed in such a way as to treat the particle homogeneously with a single effective refractive index according to the Bruggeman criterion [34]. On the other hand, in Fig. 2c and $\mathrm{d}$, an olivine core is considered covered by a carbon layer [45]. The spheres radius of Fig. 2a and $\mathrm{c}$ is $r=0.15 \mu \mathrm{m}$. On the other hand, the clusters of Fig. $2 \mathrm{~b}$ and $\mathrm{d}$ are composed by 9 spheres of different sizes with the major semi-axis $r=0.16 \mu \mathrm{m}$. In Fig. 2e, we present a model according to a field emission scanning electron microscope (FESEM) image of a silica particle clustering arranged in quenched melt spheres, shown in Fig. 2f, and collected by DUSTER [31]. The refractive index is provided by Malitson [135]. The model is composed by four spheres of different radius with the major semi-axis $r=0.23 \mu \mathrm{m}$. Figure $2 \mathrm{~g}$ represents the model of condensed $\mathrm{Ca}[\mathrm{O}]$ nanograins that are accreted onto a larger melted aggregate of tiny carbonate grains, shown in the FESEM image of Fig. 2h, and collected by DUSTER $[31,95]$. The larger sphere is calcite, and the other spheres are $\mathrm{CaO}$. The cluster model is composed by 30 spheres with the calcite refractive index provided by Ghosh, while the calcium oxide one is provided by Liu and Sieckmann [136,137]. Moreover, its major 
semi-axis has $r=0.25 \mu \mathrm{m}$. In Fig. 2i, a spherical model $(r=1.25 \mu \mathrm{m})$ of the particle Fe, Mg-rich TP2, collected by DUSTER, is shown [19]. We consider an effective refractive index obtained mixing iron (67\%) and magnesium (33\%), according to the Bruggeman criterion whose the two refractive indexes are, respectively, provided by Johnson and Christy and Hagemann [34, 138, 139]. In Fig. 2j, a spherical model of Martian hematite $(r=1 \mu \mathrm{m})$ [32]. In Fig. 2k, a spherical model of Lunar regolith $(r=1 \mu \mathrm{m})$ [33]. Figure 21 shows the model of a microscale fassaite ellipsoidal, collected by DUSTER, and shown in Fig. $2 \mathrm{~m} \mathrm{[31]} \mathrm{is} \mathrm{an}$ FESEM image. We consider an effective refractive index constructed mixing silica $(65 \%)$ and $\mathrm{CaO}(35 \%)$ according to the Bruggeman criterion [34]; the major semi-axis is $2 \mu \mathrm{m}$. All non-spherical models are oriented in such a way that their major axis is aligned with the incident light propagation direction.

The calculation of the solar radiation pressure and optical trapping forces on model particles of Fig. 2a-k is carried out with the T-matrix formalism because their size parameters fall within the range $x \approx[0.1-6]$. On the other hand, when the particle size parameter is too high (e.g., $x \geq 10$ ) such as in the fassaite microellipsoid of Fig. 21, the calculation is carried out in the ray optics approximation. For this case, we exploited the optimized computational MATLAB codes for dielectric particles provided by Callegari et al. [126] (http:// opticaltweezers.org/software/otgo-optical-tweezers-geometrical-optics/).

\section{Results on solar radiation pressure and optical trapping of dust particles}

Solar radiation pressure calculations are important to understand its relevance in optical trapping applications in space. In general, external forces, such as solar radiation pressure, can have detrimental effects on optical trapping of particles in space or the high atmosphere. Here we aim to show that T-matrix methods can be used to give accurate estimates of these effects on individual dust particles. The total radiation force, $\overline{\mathbf{F}}$, and force spectrum, $f_{s}(\lambda)$, that the Sun exerts on particles are calculated as:

$$
\begin{aligned}
\bar{F}_{s} & =\int_{\lambda} \mathrm{d} \lambda f_{s}(\lambda), \\
f_{s}(\lambda) & =\frac{i_{\odot}(\lambda)}{I_{\odot}} F_{\mathrm{rad}, s}(\lambda),
\end{aligned}
$$

where the index $s=(\|, \perp)$ specifies, respectively, the parallel and the orthogonal component of $F_{\mathrm{rad}, s}(\lambda)$ respect to the radiation incident direction, $\hat{\mathbf{k}}$. The expressions of $F_{\mathrm{rad}, s}(\lambda)$ are the wavelength-dependent optical forces calculated in the ray optics approximation [5] or in the T-matrix approach [118] according to the different models under investigation. The term $f_{s}(\lambda)$ specifies the spectral force distribution obtained scaling the computation outputs $F_{\text {rad,s }}(\lambda)$ upon the solar radiation intensity distribution $i_{\odot}(\lambda)$ normalized at the solar irradiance $I_{\odot}$, i.e., $I_{\odot}=\int_{\lambda} \mathrm{d} \lambda i_{\odot}(\lambda)=1.34 \mathrm{~kW} / \mathrm{m}^{2}$ [140]. Therefore, the considered spectrum is chosen in the range $\lambda=[0.28-2] \mu \mathrm{m}$ that is when the light emission by the Sun has the maximum intensity.

In Fig. 3, we plot the solar radiation force distributions $f_{s}(\lambda)$ (Eq. 2) for all the models presented in Fig. 2. In particular, we show the force, $f_{\|}(\lambda)$, parallel to the propagation direction as a red line, and the perpendicular one, $f_{\perp}(\lambda)$, as a blue line. Figure $3 \mathrm{a}-\mathrm{d}$ depicts the calculation on different models for the olivine-aliphatic carbon structures. Figure $3 \mathrm{e}$ is referred to the quenched melt silica, Fig. 3f the bunch-of-grape carbon Ca-rich, Fig. $3 g$ the 'TP2' sphere, and Fig. 3h the martian hematite sphere. Finally, Fig. 3i shows the fassaite ellipsoid for which the radiation force is calculated with ray optics. We note how the parallel 
Table 1 Summary table of radiation pressure and optical trapping properties

\begin{tabular}{|c|c|c|c|c|c|c|c|c|c|c|c|}
\hline MODELS & $\begin{array}{c}r \\
(\mu \mathrm{m})\end{array}$ & $\begin{array}{c}\sigma_{\text {geom }} \\
\left(\mu \mathrm{m}^{2}\right)\end{array}$ & $\overline{\boldsymbol{F}}_{\|}$ & $\overline{\boldsymbol{F}}_{\perp}$ & $\begin{array}{c}\sigma_{\text {ext }} \\
\lambda=0.83 \\
\mu \mathrm{m} \\
\left(\mu \mathrm{m}^{2}\right)\end{array}$ & $\mathrm{n}$ & $\begin{array}{r}k \\
.83 \mu \mathrm{m}\end{array}$ & $\begin{array}{l}\kappa_{\mathrm{z}} \\
\text { WA }\end{array}$ & \begin{tabular}{|c|}
\multicolumn{1}{c}{$\widetilde{\boldsymbol{\kappa}}$} \\
TER \\
$\quad(\mathrm{pN}$.
\end{tabular} & $\begin{array}{c}\kappa_{z} \\
\text { AIR/ } \\
\left(m^{-1}\right)\end{array}$ & \begin{tabular}{|c}
\multicolumn{1}{c}{$\widetilde{\kappa}$} \\
VACU \\
MM
\end{tabular} \\
\hline & 0.15 & $7.06 \cdot 10^{-2}$ & 55.45 & 0 & $3.79 \cdot 10^{-2}$ & \multirow{2}{*}{2.59} & \multirow{2}{*}{$4.73 \cdot 10^{-2}$} & \multicolumn{4}{|c|}{ No trapping } \\
\hline & 0.16 & $1.98 \cdot 10^{-2}$ & 0.93 & $3.09 \cdot 10^{-3}$ & $2.4 \cdot 10^{-4}$ & & & 6 & 25 & 2 & 14.5 \\
\hline & 0.15 & $7.06 \cdot 10^{-2}$ & 60.74 & 0 & $4.11 \cdot 10^{-2}$ & \multicolumn{2}{|c|}{ CORE } & \multicolumn{4}{|c|}{ No trapping } \\
\hline & 0.16 & $1.98 \cdot 10^{-2}$ & 0.95 & $3.08 \cdot 10^{-3}$ & $2.42 \cdot 10^{-4}$ & 2.28 & $8.83 \cdot 10^{-5}$ & 5.5 & 24.5 & 2 & 14.5 \\
\hline & 0.23 & $7.85 \cdot 10^{-2}$ & 15.78 & 0.2 & $7.96 \cdot 10^{-3}$ & 2.11 & 0 & 42.5 & 185.5 & 25.5 & 158.5 \\
\hline & 0.25 & $1.77 \cdot 10^{-1}$ & 18.2 & 1.46 & $1.15 \cdot 10^{-2}$ & \multicolumn{2}{|c|}{$\left.2.72\right|_{\mathrm{CaO}} ^{\mathrm{CaCO}_{3}} 0$} & 63 & 248.5 & 31 & 171 \\
\hline & 1.25 & 4.91 & $7.61 \cdot 10^{3}$ & 0 & 12.17 & \multirow{2}{*}{-35} & \multirow{2}{*}{20.9} & \multicolumn{4}{|c|}{ No trapping } \\
\hline & 0.05 & $7.85 \cdot 10^{-3}$ & 18.06 & 0 & $4.74 \cdot 10^{-3}$ & & & 27 & 62 & 2 & 13.5 \\
\hline & 1 & 3.14 & $5.31 \cdot 10^{3}$ & 0 & 6.84 & \multirow{2}{*}{8.03} & \multirow{2}{*}{0.14} & \multicolumn{4}{|c|}{ No trapping } \\
\hline & 0.1 & $3.14 \cdot 10^{-2}$ & 70.66 & 0 & $2.19 \cdot 10^{-2}$ & & & 148.5 & 433.5 & 33 & 151 \\
\hline & 1 & 3.14 & 2,49 & 0 & 10.85 & \multirow{2}{*}{1.68} & \multirow{2}{*}{$6.73 \cdot 10^{-2}$} & \multicolumn{4}{|c|}{ No trapping } \\
\hline & 0.1 & $3.14 \cdot 10^{-2}$ & $2.49 \cdot 10^{3}$ & 0 & $2.2 \cdot 10^{-3}$ & & & 19 & 89 & 9 & 57 \\
\hline & 2 & 12.57 & $2.56 \cdot 10^{3}$ & 0 & - & 2.42 & 0.11 & \multicolumn{4}{|c|}{ No trapping } \\
\hline
\end{tabular}

For each model particle, we show its radius or, in the case of a cluster, the radius of the smallest sphere enclosing it, $r$; the geometric cross section, $\sigma_{\text {geom }}$; the integrated optical force on the solar intensity spectrum along the parallel, $\bar{F}_{\|}$, and perpendicular direction, $\bar{F}_{\perp}$, with respect to the light propagation; the extinction cross section $\sigma_{\text {ext }}$ at the trapping wavelength $\lambda=0.83 \mu \mathrm{m}$; the real, $n$, and the imaginary part, $k$, of the refractive index at the trapping wavelength $\lambda=0.83 \mu \mathrm{m}$; and the optical trap stiffnesses along the optical axis, $\kappa_{z}$, and perpendicular to it, $\tilde{\kappa}$, in water and in air 
component, $f_{\|}$, is much larger than the perpendicular one, $f_{\perp}$. In fact, the parallel component is proportional to the particle extinction cross section, while the transverse one is related to the asymmetry parameters, $g_{i}$, related to the non-sphericity of the scatterer $[118,141,142]$. Thus, for cylindrically symmetric particles the component $f_{\perp}$ is zero (Fig. 3a, c, g-i). Figure 3b, $\mathrm{d}$, and e shows results for elongated nanoscale clusters that generally align with the incident electric field direction [12] yielding a low value of $f_{\perp}$. In Fig. 3f, we report the highly nonsymmetrical model of the bunch-of-grape carbon Ca-rich for which $f_{\perp}$ is quite strong and $\bar{F}_{\perp} \simeq 1.46 \mathrm{pN}$, a value comparable to $\bar{F}_{\|} \simeq 18.2 \mathrm{pN}$ (Table 1 ). In summary, the parallel component of the solar radiation pressure, $f_{\|}$, describes an optomechanical interaction of the solar radiation pressure with the extraterrestrial dust models in the tens of piconewton range. On the other hand, for non-spherical particles the perpendicular component of the solar radiation pressure can drive more complex transverse or rotational dynamics [143].

We now focus on the systematic characterization of optical trapping forces in optical tweezers, i.e., a single Gaussian beam focused by a high-NA objective. In our calculations, we fix the laser wavelength at $0.83 \mu \mathrm{m}$, that is a typical wavelength for optical tweezers experiments in the near-infrared, and the power $P=50 \mathrm{~mW}$. In Fig. 4, we show the three Cartesian components of the trapping force in the neighborhood of the optical tweezers paraxial nominal focus placed at the origin of the coordinate system $(x=y=z=0)$. The trapping position of the particle in the axial $z$-direction does not typically coincide with the origin because of the 'pushing' effect of the optical scattering force. To calculate the force on the particle at the equilibrium position $C_{\mathrm{eq}}=\left(x_{\mathrm{eq}}, y_{\mathrm{eq}}, z_{\mathrm{eq}}\right)$, the $z$ axial coordinate at which the axial force vanishes must firstly be found. Hence, the force plots in the transverse directions $(x, y)$ can then be calculated. In the left column of the Fig. 4, we present the extinction cross sections $\sigma_{\text {ext }}$ for the visible and near-infrared wavelength spectrum. The extinction cross section, $\sigma_{\text {ext }}$, takes into account the rate at which the energy is removed from the electromagnetic wave through scattering and the absorption, allowing us to understand how effectively trapping takes place [5]. The trapping arises when the focused incoming field generates a restoring force proportional to the particle's displacement from an equilibrium point, and that, for small displacements, behaves harmonically [7]. Therefore, trap stiffnesses are defined as:

$$
\left.\kappa_{i} \equiv \frac{\mathrm{d} F_{i}}{\mathrm{~d} x_{i}}\right|_{x_{\mathrm{eq}, i}} .
$$

We calculate optical trapping in water $\left(n_{\mathrm{m}}=1.33\right)$ with an objective $\mathrm{NA}=1.3$ (middle column of Fig. 4) and in air or vacuum $\left(n_{\mathrm{m}}=1\right)$ with NA $=0.9$ (right column of Fig. 4). We note that here we focus only on electromagnetic calculations, neglecting thermal fluctuations and hydrodynamics effects due to the surrounding medium [144]. Generally, for a given particle, optical trapping in water is stabilized by the overdamped viscous dynamics in the fluid, while in air or vacuum the underdamped dynamics might be more critical for stable optical trapping [15]. Moreover, the higher NA in water causes the equilibrium point $C_{\text {eq }}$ to be closer to the nominal focus than in air. This is confirmed by comparing the graphs in Fig. 4, central column (in water), with those of the right column (in air). The dielectric particles, such as the quenched melt silica (Fig. 4h, i), and carbonate cluster (Fig. 4k, 1) exhibit fairly stable trapping. Even the interstellar dust model of a sphere aggregate can be trapped thanks to the relatively low extinction (Fig. 4e, f). Indeed, for these model particles we are able to extrapolate the trapping constants along the axial direction, $\kappa_{z}$, and along the perpendicular direction, $\tilde{\kappa} \equiv\left(\kappa_{x}+\kappa_{y}\right) / 2$ (Table 1). On the other hand, model particles, like the interstellar dust sphere (Fig. 4b, c), or the Fe-Mg sphere (Fig. 4n, o), cannot be trapped neither in water nor in air. A similar behavior is also exhibited by the hematite sphere, regolith sphere, 
and the ellipsoidal fassaite as shown in Table 1 by observing the corresponding trapping constants. The reason is to be found in their large size (Fe-Mg sphere, hematite, regolith, and fassaite) and on their strong absorption (interstellar dust, Fe-Mg sphere, hematite, regolith, and fassaite). To further confirm, we have calculated the trapping properties of the $\mathrm{Fe}-\mathrm{Mg}$, hematite spheres by reducing their size such that $r_{\mathrm{Fe}-\mathrm{Mg}}=0.05 \mu \mathrm{m}$ and $r_{\mathrm{hem}}=0.1 \mu \mathrm{m}$. In this way, the trap stiffnesses can be extrapolated (Table 1) and a quantitative indication of $\sigma_{\text {ext }}$ is provided in Table 1. This behavior is similar to the optical trapping of metal nanoparticles that can be efficiently trapped at small size where absorption and extinction cross sections are still small in the near-infrared [145].

\section{Conclusions}

After a review on the role of interstellar, interplanetary, and planetary dust in the universe, we presented a computational study the solar radiation force and optical trapping properties for different cosmic dust particles. We showed that the radiation force exerted by the Sun is not trivially negligible: It can actively influence the dynamics of the modeled dust particles at the nano- and microscale when compared to, e.g., optical trapping forces. Furthermore, we calculated single-beam optical trapping properties for cosmic dust modeled particles both in water and in air with parameters close to typical optical tweezers experiments in the nearinfrared. We found that dielectric and weakly absorbing particles can be captured, while microparticles with stronger absorption show a scattering component of the optical force that prevents optical trapping in a standard single-beam optical tweezers. This detrimental effects can be overcome through dual-beam traps made by stationary counter-propagating laser beams $[10,146]$.

This study opens perspectives for the application of optical tweezers techniques to solar system study, e.g., cometary particles analyses including the volatile component, dust particles in the Martian atmosphere and/or on the Martian and Lunar surfaces. Such applications will also be strategic within curation facilities designed for the uncontaminated handling and preliminary characterization of extraterrestrial samples returned by space probes. The opportunity to apply optical tweezers to planetary particulate matter in the frame of curation facilities can pave the way for space applications for in situ physical chemical analyses. The accurate knowledge of the (optical, microchemical, and electrical) properties of extraterrestrial dust allows the design of an appropriate optical tweezers system for space applications, e.g., to be mounted on stratospheric platforms, onboard future cometary probes, or on landers/rovers to Mars or to the Moon.

Acknowledgements We acknowledge financial contribution from the agreement ASI-INAF n.2018-16-HH.0, Project "SPACE Tweezers". M.A.I., D.B.C., M.G.D., A.F., P.G.G., and O.M.M. acknowledge support from the MSCA ITN (ETN) Project “Active Matter”, project number 812780.

Funding Open access funding provided by Università Parthenope di Napoli within the CRUI-CARE Agreement.

Open Access This article is licensed under a Creative Commons Attribution 4.0 International License, which permits use, sharing, adaptation, distribution and reproduction in any medium or format, as long as you give appropriate credit to the original author(s) and the source, provide a link to the Creative Commons licence, and indicate if changes were made. The images or other third party material in this article are included in the article's Creative Commons licence, unless indicated otherwise in a credit line to the material. If material is not included in the article's Creative Commons licence and your intended use is not permitted by statutory 
regulation or exceeds the permitted use, you will need to obtain permission directly from the copyright holder.

To view a copy of this licence, visit http://creativecommons.org/licenses/by/4.0/.

\section{References}

1. J. Kepler, De cometis libelli tres (1619)

2. A. Ashkin, Acceleration and trapping of particles by radiation pressure. Phys. Rev. Lett. 24(4), 156 (1970)

3. A. Ashkin, Atomic-beam deflection by resonance-radiation pressure. Phys. Rev. Lett. 25(19), 1321 (1970)

4. A. Ashkin, J.M. Dziedzic, J.E. Bjorkholm, S. Chu, Observation of a single-beam gradient force optical trap for dielectric particles. Opt. Lett. 11(5), 288-290 (1986)

5. P.H. Jones, O.M. Marago, G. Volpe, Optical Tweezers: Principles and Applications (Cambridge University Press, Cambridge, 2015)

6. O.M. Maragò, P.H. Jones, P.G. Gucciardi, G. Volpe, A.C. Ferrari, Optical trapping and manipulation of nanostructures. Nat. Nanotechnol. 8(11), 807-819 (2013)

7. P. Polimeno, A. Magazzù, M.A. Iatì, F. Patti, R. Saija, C. Degli-Esposti-Boschi, M.G. Donato, P.G. Gucciardi, P.H. Jones, G. Volpe, O.M. Maragò, Optical tweezers and their applications. J. Quant. Spectrosc. Radiat. Transf. 218, 131-150 (2018)

8. D.V. Petrov, Raman spectroscopy of optically trapped particles. J. Opt. A: Pure Appl. Opt. 9(8), S139 (2007)

9. R. Gillibert, G. Balakrishnan, Q. Deshoules, M. Tardivel, A. Magazzu, M.G. Donato, O.M. Marago, M. Lamy de La Chapelle, F. Colas, F. Lagarde, P.G. Gucciardi, Raman tweezers for small microplastics and nanoplastics identification in seawater. Environ. Sci. Technol. 53(15), $9003-9013$ (2019)

10. M.G. Donato, O. Brzobohaty, S.H. Simpson, A. Irrera, A.A. Leonardi, M.J. Lo-Faro, V. Svak, O.M. Marago, P. Zemanek, Optical trapping, optical binding, and rotational dynamics of silicon nanowires in counter-propagating beams. Nano Lett. 19, 342-352 (2018)

11. F. Borghese, P. Denti, R. Saija, M.A. Iatì, Optical trapping of nonspherical particles in the T-matrix formalism. Opt. Express 15(19), 11984-11998 (2007)

12. F. Borghese, P. Denti, R. Saija, M.A. Iatì, O.M. Maragò, Radiation torque and force on optically trapped linear nanostructures. Phys. Rev. Lett. 100(16), 163903 (2008)

13. P. Zemánek, A. Jonáš, P. Jákl, J. Ježek, M. Šery, M. Liška, Theoretical comparison of optical traps created by standing wave and single beam. Opt. Commun. 220(4-6), 401-412 (2003)

14. Z. Gong, Y.-L. Pan, G. Videen, C. Wang, Optical trapping and manipulation of single particles in air: principles, technical details, and applications. J. Quant. Spectrosc. Radiat. Transf. 214, 94-119 (2018)

15. V. Svak, O. Brzobohaty, M. Šiler, P. Jákl, J. Kaňka, P. Zemánek, S.H. Simpson, Transverse spin forces and non-equilibrium particle dynamics in a circularly polarized vacuum optical trap. Nat. Commun. 9(1), 1-8 (2018)

16. H. Alali, Z. Gong, G. Videen, Y.-L. Pan, O. Muñoz, C. Wang, Laser spectroscopic characterization of single extraterrestrial dust particles using optical trapping-cavity ringdown and raman spectroscopy. J. Quant. Spectrosc. Radiat. Transf. 255, 107249 (2020)

17. P. Stysley, Laser-based optical trap for remote sampling of interplanetary and atmospheric particulate matter. Technical report, NASA (2014)

18. A. Rotundi, G.A. Baratta, J. Borg, J.R. Brucato, H. Busemann, L. Colangeli, L. d'Hendecourt, Z. Djouadi, G. Ferrini, I.A. Franchi et al., Combined micro-Raman, micro-infrared, and field emission scanning electron microscope analyses of comet 81p/wild 2 particles collected by stardust. Meteorit. Planet. Sci. 43(1-2), 367-397 (2008)

19. A. Rotundi, F.J.M. Rietmeijer, M. Ferrari, V. Della Corte, G.A. Baratta, R. Brunetto, E. Dartois, Z. Djouadi, S. Merouane, J. Borg et al., Two refractory wild 2 terminal particles from a carrot-shaped track characterized combining MIR/FIR/Raman microspectroscopy and FE-SEM/EDS analyses. Meteorit. Planet. Sci. 49(4), 550-575 (2014)

20. C. Cecchi-Pestellini, M.A. Iatì, D.A. Williams, The nature of interstellar dust as revealed by light scattering. J. Quant. Spectrosc. Radiat. Transfer 113(18), 2310-2320 (2012)

21. B.A. McGuire, 2018 census of interstellar, circumstellar, extragalactic, protoplanetary disk, and exoplanetary molecules. Astrophys. J. Suppl. 239, 17 (2018)

22. H.P. Gail, E. Sedlmayr, Physics and Chemistry of Circumstellar Dust Shells, Cambridge Astrophysics. (Cambridge University Press, Cambridge, 2014) 
23. A. Sarangi, I. Cherchneff, Condensation of dust in the ejecta of type II-P supernovae. Astron. Astrophys. 575, A95 (2015)

24. C. Gall, J. Hjorth, D. Watson, E. Dwek, J.R. Maund, O. Fox, G. Leloudas, D. Malesani, A.C. Day-Jones, Rapid formation of large dust grains in the luminous supernova 2010j1. Nature 511(7509), 326-329 (2014)

25. A. Giannetti, S. Leurini, C. König, J.S. Urquhart, T. Pillai, J. Brand, J. Kauffmann, F. Wyrowski, K.M. Menten, Galactocentric variation of the gas-to-dust ratio and its relation with metallicity. Astron. Astrophys. 606, L12 (2017)

26. T. Zafar, D. Watson, The metals-to-dust ratio to very low metallicities using GRB and QSO absorbers; extremely rapid dust formation. Astron. Astrophys. 560, A26 (2013)

27. C. Gall, J. Hjorth, A.C. Andersen, Production of dust by massive stars at high redshift. Astron. Astrophys. Rev. 19, 43 (2011)

28. L. Mattsson, A. De Cia, A.C. Andersen, T. Zafar, On the (in)variance of the dust-to-metals ratio in galaxies. Mon. Not. R. Astron. Soc. 440, 1562-1570 (2014)

29. M. Bocchio, A.P. Jones, J.D. Slavin, A re-evaluation of dust processing in supernova shock waves. Astron. Astrophys. 570, A32 (2014)

30. B.T. Draine, Interstellar Dust Models and Evolutionary Implications. In T. Henning, E. Grün, J. Steinacker, editors, Cosmic Dust - Near and Far, volume 414 of Astronomical Society of the Pacific Conference Series, page 453, (2009)

31. F.J.M. Rietmeijer, V. Della-Corte, M. Ferrari, A. Rotundi, R. Brunetto, Laboratory analyses of meteoric debris in the upper stratosphere from settling bolide dust clouds. Icarus 266, 217-234 (2016)

32. F. Oliva, E. D’Aversa, G.L. Liberti, G. Sindoni, F. Altieri, E. Castelli, A database of aerosols and gases coefficients for VIS-NIR radiative transfer in the solar system planetary atmospheres. Planet. Space Sci. 166, 101-109 (2019)

33. W.G. Egan, T. Hilgeman, Optical constants for terrestrial analogs of lunar materials. Astron. J. 78, 799 (1973)

34. C.F. Bohren, D.R. Huffman, Absorption and Scattering of Light by Small Particles (Wiley, Hoboken, 2008)

35. E.L. Fitzpatrick, D. Massa, K.D. Gordon, R. Bohlin, G.C. Clayton, An analysis of the shapes of interstellar extinction curves. vii. Milky way spectrophotometric optical-through-ultraviolet extinction and its rdependence. Astrophys. J. 886, 108 (2019)

36. N.A. Reddy, A.E. Shapley, M. Kriek, C.C. Steidel, I. Shivaei, R.L. Sanders, B. Mobasher, A.L. Coil, B. Siana, W.R. Freeman et al., The MOSDEF survey: the first direct measurements of the nebular dust attenuation curve at high redshift. Astrophys. J. 902, 123 (2020)

37. D.A. Williams, C. Cecchi-Pestellini, The Chemistry of Cosmic Dust (Royal Society of Chemistry, London, 2015)

38. K.A.K. Gadallah, H. Mutschke, C. Jäger, UV irradiated hydrogenated amorphous carbon (HAC) materials as a carrier candidate of the interstellar UV bump at $217.5 \mathrm{~nm}$. Astron. Astrophys. 528, A56 (2011)

39. A.G.G.M. Tielens, The Physics and Chemistry of the Interstellar Medium (Cambridge University Press, Cambridge, 2005)

40. S.A. Sandford, L.J. Allamandola, A.G.G.M. Tielens, K. Sellgren, M. Tapia, Y. Pendleton, The interstellar $\mathrm{CH}$ stretching band near 3.4 microns-constraints on the composition of organic material in the diffuse interstellar medium. Astrophys. J. 371, 607-620 (1991)

41. B.T. Draine, Scattering by interstellar dust grains. ii. X-rays. Astrophys. J. 598(2), 1026 (2003)

42. A. Leger, J.L. Puget, Identification of the unidentified IR emission features of interstellar dust?? Astron. Astrophys. 500, 279-282 (1984)

43. W.W. Duley, D.A. Williams, Excitation of the aromatic infrared emission bands: chemical energy in hydrogenated amorphous carbon particles? Astrophys. J. Lett. 737, L44 (2011)

44. E. Dartois, T.R. Geballe, T. Pino, A.T. Cao, A. Jones, D. Deboffle, V. Guerrini, Ph Bréchignac, L. D'Hendecourt, IRAS 08572+3915: constraining the aromatic versus aliphatic content of interstellar HACs. Astron. Astrophys. 463(2), 635-640 (2007)

45. M.A. Iatì, R. Saija, F. Borghese, P. Denti, C. Cecchi-Pestellini, D.A. Williams, Stratified dust grains in the interstellar medium-I. An accurate computational method for calculating their optical properties. Month. Notices R. Astron. Soc. 384(2), 591-598 (2008)

46. C. Cecchi-Pestellini, A. Cacciola, M.A. Iatì, R. Saija, F. Borghese, P. Denti, A. Giusto, D.A. Williams, Stratified dust grains in the interstellar medium-ii. Time-dependent interstellar extinction. Month. Notices R. Astron. Soc. 408(1), 535-541 (2010)

47. M.F. Nieva, N. Przybilla, Carbon abundances of early b-type stars in the solar vicinity-Non-LTE lineformation for C II/III/IV and self-consistent atmospheric parameters. Astron. Astrophys. 481, 199-216 (2008) 
48. E.B. Jenkins, A unified representation of gas-phase element depletions in the interstellar medium. Astrophys. J. 700, 1299 (2009)

49. B.T. Draine, B. Hensley, Magnetic nanoparticles in the interstellar medium: emission spectrum and polarization. Astrophys. J. 765, 159 (2013)

50. D.C.B. Whittet, Oxygen depletion in the interstellar medium: implications for grain models and the distribution of elemental oxygen. Astrophys. J. 710(2), 1009-1016 (2010)

51. M. Asplund, N. Grevesse, A.J. Sauval, P. Scott, The chemical composition of the sun. Ann. Rev. Astron. Astrophys. 47, 481-522 (2009)

52. F.C. Gillett, W.J. Forrest, Spectra of the Becklin-Neugebauer point source and the Kleinmann-low nebula from 2.8 to 13.5 microns. Astrophys. J. 179, 483 (1973)

53. A.C.A. Boogert, P.A. Gerakines, D.C.B. Whittet, Observations of the icy universe. Ann. Rev. Astron. Astrophys. 53, 541-581 (2015)

54. G.A. Baratta, D. Chaput, H. Cottin, L. Fernandez Cascales, M.E. Palumbo, G. Strazzulla, Ion bombardment of cometary ices analogues: production of organic samples for the EXPOSE-R2 mission on the International Space Station. Eur. Planet. Sci. Congress 9, EPSC2014-46 (2014)

55. A. Ciaravella, A. Jiménez-Escobar, C. Cecchi-Pestellini, C.H. Huang, N.E. Sie, G.M. Muñoz Caro, Y.J. Chen, Synthesis of complex organic molecules in soft X-ray irradiated ices. Astrophys. J. 879, 21 (2019)

56. J.A. Nuth, J.H. Hecht, Signatures of aging silicate dust. Astrophys. Space Sci. 163(1), 79-94 (1990)

57. F.J.M. Rietmeijer, J.A. Nuth, Petrologic constraints on amorphous and crystalline magnesium silicates: dust formation and evolution in selected Herbig Ae/Be systems. Astrophys. J. 771, 34 (2013)

58. A. Ciaravella, C. Cecchi-Pestellini, Y.J. Chen, G.M. Muñoz Caro, C.H. Huang, A. Jiménez-Escobar, A.M. Venezia, soft X-ray irradiation of silicates: implications for dust evolution in protoplanetary disks. Astrophys. J. 828, 29 (2016)

59. A. Juhász, J. Bouwman, Th Henning, B. Acke, M.E. van den Ancker, G. Meeus, C. Dominik, M. Min, A.G.G.M. Tielens, L.B.F.M. Waters, Dust evolution in protoplanetary disks around Herbig Ae/Be stars-the spitzer view. Astrophys. J. 721, 431-455 (2010)

60. T.P. Stecher, B. Donn, On graphite and interstellar extinction. Astrophys. J. 142, 1681 (1965)

61. J.S. Mathis, G. Whiffen, Composite interstellar grains. Astrophys. J. 341, 808 (1989)

62. E.L. Wright, Long-wavelength absorption by fractal dust grains. Astrophys. J. 320, 818 (1987)

63. Ch. Joblin, A. Léger, P. Martin, Contribution of polycyclic aromatic hydrocarbon molecules to the interstellar extinction curve. Astrophys. J. 393, L79-L82 (1992)

64. R. Papoular, J. Conard, O. Guillois, I. Nenner, C. Reynaud, J.-N. Rouzaud, A comparison of solid-state carbonaceous models of cosmic dust. Astron. Astrophys. 315, 222-236 (1996)

65. T.M. Steel, W.W. Duley, A 217.5 nanometer absorption feature in the spectrum of small silicate particles. Astrophys. J. 315, 337 (1987)

66. A. Higa, T. Oshiro, Y. Saida, M. Yamazato, M. Toguchi, Correlation between properties and hydrogen concentration of ac: $\mathrm{H}$ films prepared by RF magnetron sputtering. New Diamond Front. Carbon Technol. 16(5), 245-253 (2006)

67. N. Altobelli, F. Postberg, K. Fiege, M. Trieloff, H. Kimura, V.J. Sterken, H.W. Hsu, J. Hillier, N. Khawaja, G. Moragas-Klostermeyer, J. Blum, M. Burton, R. Srama, S. Kempf, E. Gruen, Flux and composition of interstellar dust at Saturn from Cassini's cosmic dust analyzer. Science 352(6283), 312-318 (2016)

68. A.E. Rubin, J.N. Grossman, Meteorite and meteoroid: new comprehensive definitions. Meteorit. Planet. Sci. 45(1), 114-122 (2010)

69. D. Nesvorny, D. Vokrouhlicky, W.F. Bottke, M. Sykes, Physical properties of asteroid dust bands and their sources. Icarus 181(1), 107-144 (2006)

70. G.J. Flynn, D.D. Durda, L.E. Sandel, J.W. Kreft, M.M. Strait, Dust production from the hypervelocity impact disruption of the Murchison hydrous CM2 meteorite: implications for the disruption of hydrous asteroids and the production of interplanetary dust. Planet. Space Sci. 57(2), 119-126 (2009)

71. M. Fulle, J. Blum, A. Rotundi, B. Gundlach, C. Güttler, V. Zakharov, How comets work: nucleus erosion versus dehydration. Mon. Not. R. Astron. Soc. 493(3), 4039-4044 (2020)

72. C. Güttler, T. Mannel, A. Rotundi, S. Merouane, M. Fulle, D. Bockelée-Morvan, J. Lasue, A.C. LevasseurRegourd, J. Blum, G. Naletto et al., Synthesis of the morphological description of cometary dust at comet 67P/Churyumov-Gerasimenko. Astron. Astrophys. 630, A24 (2019)

73. J. Blum, B. Gundlach, M. Krause, M. Fulle, A. Johansen, J. Agarwal, I. Von Borstel, X. Shi, X. Hu, M.S. Bentley et al., Evidence for the formation of comet 67P/Churyumov-Gerasimenko through gravitational collapse of a bound clump of pebbles. Month. Notices R. Astrono. Soc. 469(Suppl-2), S755-S773 (2017)

74. B. Peucker-Ehrenbrink, Accretion of extraterrestrial matter during the last 80 million years and its effect on the marine osmium isotope record. Geochim. Cosmochim. Acta 60(17), 3187-3196 (1996)

75. S.A. Sandford, C. Engrand, A. Rotundi, Organic matter in cosmic dust. Elements 12(3), 185-189 (2016) 
76. S.G. Love, D.E. Brownlee, A direct measurement of the terrestrial mass accretion rate of cosmic dust. Science 262(5133), 550-553 (1993)

77. E. Grün, H.A. Zook, H. Fechtig, R.H. Giese, Collisional balance of the meteoritic complex. Icarus 62(2), 244-272 (1985)

78. S.J. Kortenkamp, S.F. Dermott, D. Fogle, K. Grogan, Sources and orbital evolution of interplanetary dust accreted by earth, in Accretion of Extraterrestrial Matter Throughout Earth's History. ed. by B. Peucker-Ehrenbrink, B. Schmitz (Springer, Berlin, 2001), pp. 13-30

79. D. Nesvorny, P. Jenniskens, H.F. Levison, W.F. Bottke, D. Vokrouhlicky, M. Gounelle, Cometary origin of the zodiacal cloud and carbonaceous micrometeorites. Implications for hot debris disks. Astrophys. J. 713(2), 816 (2010)

80. C. Cordier, L. Folco, Oxygen isotopes in cosmic spherules and the composition of the near earth interplanetary dust complex. Geochim. Cosmochim. Acta 146, 18-26 (2014)

81. M.D. Suttle, L. Folco, The extraterrestrial dust flux: size distribution and mass contribution estimates inferred from the transantarctic mountains (TAM) micrometeorite collection. J. Geophys. Res.: Planets 125(2), e2019JE006241 (2020)

82. M.D. Suttle, Z. Dionnet, I. Franchi, L. Folco, J. Gibson, R.C. Greenwood, A. Rotundi, A. King, S.S. Russell, Isotopic and textural analysis of giant unmelted micrometeorites-identification of new material from intensely altered 16o-poor water-rich asteroids. Earth Plant. Sci. Lett. 546, 116444 (2020)

83. Z. Dionnet, M.D. Suttle, A. Longobardo, A. Rotundi, L. Folco, V. Della-Corte, A. King, X-ray computed tomography: morphological and porosity characterization of giant antarctic micrometeorites. Meteorit. Planet. Sci. 55(7), 1581-1599 (2020)

84. V. Della-Corte, P. Palumbo, A. Rotundi, S. De Angelis, F.J.M. Rietmeijer, E. Bussoletti, A. Ciucci, M. Ferrari, V. Galluzzi, E. Zona, In situ collection of refractory dust in the upper stratosphere: the duster facility. Space Sci. Rev. 169(1-4), 159-180 (2012)

85. F.J.M. Rietmeijer, The earliest chemical dust evolution in the solar nebula. Geochemistry 62(1), 1-45 (2002)

86. Z. Dionnet, R. Brunetto, A. Aléon-Toppani, S. Rubino, D. Baklouti, F. Borondics, A.-C. Buellet, Z. Djouadi, A. King, T. Nakamura et al., Combining IR and x-ray microtomography data sets: Application to Itokawa particles and to Paris meteorite. Meteorit. Planet. Sci. 55, 1645-1664 (2020)

87. V. Della-Corte, A. Rotundi, M. Fulle, S. Ivanovski, S.F. Green, F.J.M. Rietmeijer, L. Colangeli, P. Palumbo, R. Sordini, M. Ferrari et al., 67P/CG inner coma dust properties from 2.2 au inbound to 2.0 au outbound to the sun. Month. Notices R. Astron. Soc. 462(Suppl-1), S210-S219 (2016)

88. M. Fulle, V. Della-Corte, A. Rotundi, P. Weissman, A. Juhasz, K. Szego, R. Sordini, M. Ferrari, S. Ivanovski, F. Lucarelli et al., Density and charge of pristine fluffy particles from comet 67P/ChuryumovGerasimenko. Astrophys. J. Lett. 802(1), L12 (2015)

89. A. Rotundi, H. Sierks, V. Della-Corte, M. Fulle, P.J. Gutierrez, L. Lara, C. Barbieri, P.L. Lamy, R. Rodrigo, D. Koschny et al., Dust measurements in the coma of comet 67P/Churyumov-Gerasimenko inbound to the sun. Science 347(6220), aaa3905-6 (2015)

90. L. Folco, C. Cordier, Micrometeorites. EMU notes in mineralogy. In Planetary Mineralogy M. R. Lee \& H. Leroux (Eds.), volume 15, pages 253-297. European Mineralogical Union, The Mineralogical Society of Great Britain and Ireland (2015)

91. M. Van Ginneken, L. Folco, C. Cordier, P. Rochette, Chondritic micrometeorites from the transantarctic mountains. Meteorit. Planet. Sci. 47(2), 228-247 (2012)

92. T. Kohout, A. Kallonen, J.-P. Suuronen, P. Rochette, A. Hutzler, J. Gattacceca, D.D. Badjukov, R. Skala, V. Böhmová, J. Čuda, Density, porosity, mineralogy, and internal structure of cosmic dust and alteration of its properties during high-velocity atmospheric entry. Meteorit. Planet. Sci. 49, 1157-1170 (2014)

93. P. Rochette, L. Folco, C. Suavet, M. Van Ginneken, J. Gattacceca, N. Perchiazzi, R. Braucher, R.P. Harvey, Micrometeorites from the transantarctic mountains. Proc. Natl. Acad. Sci. 105(47), 18206-18211 (2008)

94. C. Suavet, P. Rochette, M. Kars, J. Gattacceca, L. Folco, R.P. Harvey, Statistical properties of the transantarctic mountains (TAM) micrometeorite collection. Polar Sci. 3(2), 100-109 (2009)

95. V. Della-Corte, F.J.M. Rietmeijer, A. Rotundi, M. Ferrari, P. Palumbo, Meteoric CAO and carbon smoke particles collected in the upper stratosphere from an unanticipated source. Tellus B: Chem. Phys. Meteorol. 65(1), 20174 (2013)

96. A. Rotundi, G. Ferrini, G.A. Baratta, M.E. Palumbo, E. Palomba, L. Colangeli, Combined micro-infrared (IR) and micro-Raman measurements on stratospheric interplanetery dust particles. ESASP 643, 149153 (2007)

97. R. Brunetto, J. Borg, E. Dartois, F.J.M. Rietmeijer, F. Grossemy, C. Sandt, L. Le Sergeant-d'Hendecourt, A. Rotundi, P. Dumas, Z. Djouadi et al., Mid-IR, Far-IR, Raman micro-spectroscopy, and FESEM-EDX study of IDP L2021C5: Clues to its origin. Icarus 212(2), 896-910 (2011) 
98. V. Della Corte, A. Rotundi, V. Zakharov, S. Ivanovski, P. Palumbo, M. Fulle, A. Longobardo, Z. Dionnet, V. Liuzzi, M. Salatti, Giada microbalance measurements on board Rosetta: submicrometerto micrometer-sized dust particle flux in the coma of comet 67P/Churyumov-Gerasimenko. Astron. Astrophys. 630, A25 (2019)

99. V. Della-Corte, R. Sordini, M. Accolla, M. Ferrari, S. Ivanovski, A. Rotundi, F.J.M. Rietmeijer, M. Fulle, E. Mazzotta-Epifani, P. Palumbo et al., Giada-grain impact analyzer and dust accumulator-onboard Rosetta spacecraft: extended calibrations. Acta Astronautica 126, 205-214 (2016)

100. V.V. Zakharov, S.L. Ivanovski, J.-F. Crifo, V. Della-Corte, A. Rotundi, M. Fulle, Asymptotics for spherical particle motion in a spherically expanding flow. Icarus 312, 121-127 (2018)

101. S.F. Dermott, T.J.J. Kehoe, K. Grogan, D.D. Durda, S. Jayaraman, S.J. Kortenkamp, M.C. Wyatt, Orbital evolution of interplanetary dust, in Interplanetary Dust. ed. by E. Grun, B.A.S. Gustafson, S. Dermott, H. Fechtig (Springer, Berlin, 2001), pp. 569-639

102. P. Lucey, R.L. Korotev, J.J. Gillis, L.A. Taylor, D. Lawrence, B.A. Campbell, R. Elphic, B. Feldman, L.L. Hood, D. Hunten et al., Understanding the lunar surface and space-moon interactions. Rev. Mineral. Geochem. 60, 83-219 (2006)

103. D.S. McKay, G. Heiken, A. Basu, G. Blanford, S. Simon, R. Reedy, B.M. French, J. Papike, The lunar regolith, in Lunar Sourcebook: A User's Guide to the Moon. ed. by G.H. Heiken, D.T. Vaniman, B.M. French (Cambridge University Press, Cambridge, 1991), pp. 285-356

104. W.D. III Carrier, G.R. Olhoeft, W. Mendell, Physical Properties of the Lunar Surface, vol. 475-594 (Cambridge University Press, Cambridge, 1991)

105. E.C.T. Chao, J.A. Boreman, J.A. Minkin, O.B. James, G.A. Desborough, Lunar glasses of impact origin: physical and chemical characteristics and geologic implications. J. Geophys. Res. 75(35), 7445-7479 (1970)

106. O.B. Toon, J.B. Pollack, C. Sagan, Physical properties of the particles composing the Martian dust storm of 1971-1972. Icarus 30(4), 663-696 (1977)

107. B.C. Clark, A.K. Baird, R.J. Weldon, D.M. Tsusaki, L. Schnabel, M.P. Candelaria, Chemical composition of Martian fines. J. Geophys. Res.: Solid Earth 87(B12), 10059-10067 (1982)

108. C.N. Foley, T. Economou, R.N. Clayton, Final chemical results from the mars pathfinder alpha proton X-ray spectrometer. J. Geophys. Res.: Planets 108(E12), 8095 (2003)

109. R.V. Morris, G. Klingelhoefer, C. Schroder, D.S. Rodionov, A. Yen, D.W. Ming, P.A. De Souza, T. Wdowiak, I. Fleischer, R. Gellert, B. Bernhardt, Mossbauer mineralogy of rock, soil, and dust at meridiani planum, mars: opportunity's journey across sulfate-rich outcrop, basaltic sand and dust, and hematite lag deposits. J. Geophys. Res.: Planets 111(E12), E12S15 (2006)

110. A.S. Yen, R. Gellert, C. Schröder, R.V. Morris, J.F. Bell, A.T. Knudson, B.C. Clark, D.W. Ming, J.A. Crisp, R.E. Arvidson et al., An integrated view of the chemistry and mineralogy of Martian soils. Nature 436(7047), 49-54 (2005)

111. H.Y. McSween Jr., I.O. McGlynn, A.D. Rogers, Determining the modal mineralogy of Martian soils. J. Geophys. Res.: Planets 115(E7), E00F12 (2010)

112. D.L. Bish, D.F. Blake, D.T. Vaniman, S.J. Chipera, R.V. Morris, D.W. Ming, A.H. Treiman, P. Sarrazin, S.M. Morrison, R.T. Downs et al., X-ray diffraction results from mars science laboratory: mineralogy of rocknest at gale crater. Science 341(6153), 1238932 (2013)

113. H. Chen-Chen, S. Perez-Hoyos, A. Sánchez-Lavega, Dust particle size, shape and optical depth during the 2018/my34 Martian global dust storm retrieved by MSL curiosity rover navigation cameras. Icarus 354, 114021 (2020)

114. F. Oliva, A. Geminale, E. D’Aversa, F. Altieri, G. Bellucci, F.G. Carrozzo, G. Sindoni, D. Grassi, Properties of a Martian local dust storm in Atlantis chaos from OMEGA/MEX data. Icarus 300, 1-11 (2018)

115. M.J. Wolff, M.D. Smith, R.T. Clancy, R. Arvidson, M. Kahre, F. Seelos, S. Murchie, H. Savijärvi, Wavelength dependence of dust aerosol single scattering albedo as observed by the compact reconnaissance imaging spectrometer. J. Geophys. Res.: Planets 114(E2), E00D04 (2009)

116. M.J. Wolff, R.T. Clancy, J.D. Goguen, M.C. Malin, B.A. Cantor, Ultraviolet dust aerosol properties as observed by Marci. Icarus 208(1), 143-155 (2010)

117. F. Esposito, R. Molinaro, C.I. Popa, C. Molfese, F. Cozzolino, L. Marty, K. Taj-Eddine, G. Di Achille, G. Franzese, S. Silvestro et al., The role of the atmospheric electric field in the dust-lifting process. Geophys. Res. Lett. 43, 5501-5508 (2016)

118. F. Borghese, P. Denti, R. Saija, Scattering from Model Nonspherical Particles: Theory and Applications to Environmental Physics (Springer, Berlin, 2007)

119. M.I. Mishchenko, L.D. Travis, A.A. Lacis, Scattering, Absorption, and Emission of Light by Small Particles (Cambridge University Press, Cambridge, 2002) 
120. P. Polimeno, R. Saija, C. Degli-Esposti-Boschi, O.M. Maragò, M.A. Iatì, Optical forces in the t-matrix formalism. Atti della Accademia Peloritana dei Pericolanti-Classe di Scienze Fisiche, Matematiche e Naturali 97(1), 2 (2019)

121. P.C. Chaumet, M. Nieto-Vesperinas, Time-averaged total force on a dipolar sphere in an electromagnetic field. Opt. Lett. 25(15), 1065-1067 (2000)

122. J.R. Arias-González, M. Nieto-Vesperinas, Optical forces on small particles: attractive and repulsive nature and plasmon-resonance conditions. JOSA A 20(7), 1201-1209 (2003)

123. S. Albaladejo, M.I. Marqués, M. Laroche, J.J. Sáenz, Scattering forces from the curl of the spin angular momentum of a light field. Phys. Rev. Lett. 102(11), 113602 (2009)

124. A. Ashkin, Forces of a single-beam gradient laser trap on a dielectric sphere in the ray optics regime. Biophys. J. 61(2), 569-582 (1992)

125. M. Born, E. Wolf, Principles of Optics: Electromagnetic Theory of Propagation, Interference and Diffraction of Light (Cambridge University Press, Cambridge, 1999)

126. A. Callegari, M. Mijalkov, A.B. Gököz, G. Volpe, Computational toolbox for optical tweezers in geometrical optics. JOSA B 32(5), B11-B19 (2015)

127. P.C. Waterman, Symmetry, unitarity, and geometry in electromagnetic scattering. Phys. Rev. D 3(4), 825 (1971)

128. F. Borghese, P. Denti, G. Toscano, O.I. Sindoni, An addition theorem for vector Helmholtz harmonics. J. Math. Phys. 21(12), 2754-2755 (1980)

129. E. Fucile, P. Denti, F. Borghese, R. Saija, O.I. Sindoni, Optical properties of a sphere in the vicinity of a plane surface. Journal of the Optical Society of America A 14, 1505-1514 (1997)

130. A. Ishimaru, Wave propagation and scattering in random media and rough surfaces. Proc. IEEE 79(10), 1359-1366 (1991)

131. M.A. Iatì, A. Giusto, R. Saija, F. Borghese, P. Denti, C. Cecchi-Pestellini, S. Aiello, Optical properties of composite interstellar grains: a morphological analysis. Astrophys. J. 615(1), 286 (2004)

132. R. Saija, M.A. Iatì, P. Denti, F. Borghese, A. Giusto, O.I. Sindoni, Efficient light-scattering calculations for aggregates of large spheres. Appl. Opt. 42(15), 2785-2793 (2003)

133. B.T. Draine, H.M. Lee, Optical properties of interstellar graphite and silicate grains. Astrophys. J. 285, 89-108 (1984)

134. E.D. Palik, Handbook of Optical Constants of Solids, vol. 3 (Academic Press, Cambridge, 1998)

135. I.H. Malitson, Interspecimen comparison of the refractive index of fused silica. JOSA 55, 1205-1209 (1965)

136. G. Ghosh, Dispersion-equation coefficients for the refractive index and birefringence of calcite and quartz crystals. Opt. Commun. 163(1-3), 95-102 (1999)

137. C.J. Liu, E.F. Sieckmann, Refractive index of calcium oxide. J. Appl. Phys. 37(6), 2450-2452 (1966)

138. P.B. Johnson, R.-W. Christy, Optical constants of the noble metals. Phys. Rev. B 6(12), 4370 (1972)

139. H.J. Hagemann, W. Gudat, C. Kunz, Desy Report Sr-74/7 (Hamburg, Germany, 1974)

140. A.S.T.M. Standard, G173-03-standard tables for reference solar spectral irradiances: Direct normal and hemispherical on 37 tilted surface. Ann. Book ASTM Standards 2003, 14 (2012)

141. M.I. Mishchenko, Radiation force caused by scattering, absorption, and emission of light by nonspherical particles. J. Quant. Spectrosc. Radiat. Transfer 70(4-6), 811-816 (2001)

142. R. Saija, M.A. Iatì, A. Giusto, P. Denti, F. Borghese, Transverse components of the radiation force on nonspherical particles in the T-matrix formalism. J. Quant. Spectrosc. Radiat. Transfer 94(2), 163-179 (2005)

143. J. Klačka, M. Kocifaj, Motion of nonspherical dust particle under the action of electromagnetic radiation. J. Quant. Spectrosc. Radiat. Transfer 70(4-6), 595-610 (2001)

144. G. Volpe, G. Volpe, Simulation of a Brownian particle in an optical trap. Am. J. Phys. 81(3), 224-230 (2013)

145. V. Amendola, R. Pilot, M. Frasconi, O.M. Maragò, M.A. Iatì, Surface plasmon resonance in gold nanoparticles: A review. J. Phys.: Condens. Matter 29, 203002 (2017)

146. P. Zemánek, A. Jonáš, M. Liška, Simplified description of optical forces acting on a nanoparticle in the gaussian standing wave. JOSA A 19(5), 1025-1034 (2002) 